\title{
Networks formation to assist decision making
}

\author{
David Goldbaum*
}

April 29, 2016

\begin{abstract}
This paper examines network formation among a connected population with a preference for conformity and leadership. Agents build stable personal relationships to achieve coordinated actions. The network serves as a repository of collective experiences so that cooperation can emerge from simple, myopic, self-serving adaptation to recent events despite the competing impulses of conformity and leadership and despite limiting individuals to only local information. Computational analysis reveals how behavioral tendencies impact network formation and identifies locally stable disequilibrium structures.
\end{abstract}

Keywords: Leader, Dynamic Network, Payoff interdependence, Social Interaction, Simulation (JEL Codes: D85, D71, C71)

\footnotetext{
*Economics Discipline Group, University of Technology Sydney, PO Box 123 Broadway, NSW 2007 Australia, david.goldbaum@uts.edu.au
} 
One does not become a guru by accident.

James Fenton ${ }^{1}$

\section{Introduction}

Sellers stylishly enhance functionally equivalent product offerings, thereby appealing to buyers' subjective tastes. The prevalence of experts offering guidance in subjective goods suggest social influences play a role in decision making. In a number of settings, decision makers prefer conformity. What constitutes an expert of a subjective good may simply be someone with an audience. ${ }^{2}$ Social influence invites strategies played over social position, rather than over product choice, in order to achieve conformity, particularly if the preference for conformity is enhanced by the appearance of influence over the decisions of others. ${ }^{3}$

With social interactions, when to act is as much an element of strategy as how to act. Social positioning cannot arise spontaneously. Repeated decisions, like those offered with each new fashion season, offer opportunity for social repositioning and reputation. This paper investigates the evolution in social positioning over time via a network of links as players seek to achieve conformity and leadership.

A utility-based desire for conformity can be modeled as arising out of a social component, such as in Brock and Durlauf (2001). With modification, the social component also provides a utility reward to early adopters based on the population of matching subsequent adopters, as in Goldbaum (2016).

The inability to employ a consistent language with which to refer to the ever-changing targets of coordination makes the product-space strategies employed in the coordination game of Crawford and Haller (1990) unavailable to the agents in the current model. A network of reliable social connections is the foundation upon which the agents develop strategies to facilitate coordination.

The social network has long been recognized as a vehicle for coordinating activities. Social networks generate by-directional interactions between local and aggregate behavior. For Schelling (1971), Schelling (1973), and Katz and Shapiro (1985) the individual discrete decisions are sensitive to the information available through network connections, influencing which new products or technologies are adopted. Because of social interactions, products that garner no particular consumer preference can grow to dominate consumer choice.

\footnotetext{
${ }^{1}$ Columbia World of Quotations. Columbia University Press, 1996. http://quotes.dictionary.com/Onedoesnotbecomeagurubyaccident (accessed: March 17, 2011).

${ }^{2} \mathrm{~A}$ guru is "a teacher who attracts disciples or followers." An expert is "a person who has special skill or knowledge in some particular field; specialist; authority." (Dictionary.com, 2016) Maybe more guru than expert.

3 "Wine geeks just love bragging rights. They get kudos from their peers when they get a high-score wine first or get it cheaper" (Bialek quoted in Los Angeles Magazine, 1998(Dec)). The social component of acting in advance of a trend is highlighted as distinct from a simple desire to adopt products when they are new, a desire that is not necessarily socially derived.
} 
A network is also a vehicle for information transmission as, for example, the Ellison and Fudenberg (1995) use of an undirected network and in the directed network of Dutta and Jackson (2000).

Consensus and information transmission are at the heart of the DeGroot (1974) social learning model. The social network is a conduit of information by which populations update beliefs in an effort to eliminate private biases. The ability of privately informed linked individuals to reach consensus on the truth depends on the structure of the network. As identified by Corazzini et al. (2012), individuals with outsized influence facilitate convergence while impeding the emergence of the truth unless they are also well-connected gatherers of information.

The Acemoglu et al. (2013) extension to the social learning model finds that a population subset which retains its private opinion impacts beliefs and convergence, as one might expect from an opinion leader. Acemoglu et al. (2010) injects a time element with agents pressed to act on their beliefs by a penalty for delaying adoption. Arifovic et al. (2015) identifies a preference for consensus affecting the updating of beliefs. Inherent in the social learning models is an intrinsic truth that each agent wishes to learn. As a Bayesian or employing some non-Bayesian alternative, they look to make use of every bit of information available to them to uncover the truth. In the current setting, there is no truth, only a social construct. The social learning mechanism of gathering and aggregating information does not serve the individual's objectives for conformity and influence.

Ali and Kartik (2012) introduces a preference over the actions of others to the model of observational learning. Borrowing one of the authors' examples, the model applies to agents making political contributions to gain favor from the winning candidate. With learning, early contributors influence the target of subsequent contributions, affecting election outcomes. Later contributors have an informational advantage about which candidate is likely to win based on prior support. Following the original model of Banerjee (1992), Ali and Kartik (2012) have agents decide sequentially. Pushing the scenario beyond the original example, it would be reasonable to allow the politician to place grater value on early contributions, knowing the potential influential impact it can have on downstream contributors. This creates a tradeoff between contributing early or delaying. Freeing contributors to choose the timing of a contribution increases the uncertainty, particularly when contributions can be made simultaneously, as contributors cannot know the value of their contribution on subsequent decision makers.

Achieving conformity requires forming a purposeful network for transmitting and receiving information. In the developed model, agents establish one-sided directed links in order to receive information on the adoption decision of the link's target as a means to generate coordination. New linking decisions are made 
simultaneously for the current adoption decision. Each agent evaluates her decision ex post based on the outcome versus counterfactual payoffs offered by other linking options and of choosing autonomously. This ex post evaluation informs the agent's linking decision for the next adoption decision. This paper investigates environmental influences on the ability of the population to develop a coordinating network. Also explored are individual characteristics as they affect individual positioning in the emergent network.

The simultaneous, rather than sequential, linking decisions avoids exogenously imposed advantages in positioning. This precludes the network formation process based on ordered sequential linking decisions of Jackson and Wolinsky (1996), Watts (2001) and related works. Bala and Goyal (2000) allows myopic best-response one-sided link formation by randomly chosen agents with full knowledge of the network. The current investigation envisions the game played by a potentially large population, where knowledge of the full network is unknowable or impractical. Agents have access to aggregate information concerning previous realizations of popularity but only local knowledge about individual adoptions and payoffs. Nonetheless, the leader-centric star network (or, more properly given limited linking options, a leader-centered hub) is the natural target structure for its ability to coordinate activity.

Like Chang and Harrington (2007), a symbiotic relationship emerges according to roles individuals find themselves in. Agents adapt to their changing local environment in a payoff improving manner. Simulations characterize the behavior of the population. Individual strategies are modeled as evolving according to the Experience Weighted Attractor (EWA) of Camerer and Ho (1999). Agents employ actions probabilistically according to a nested logit model of Hausman and McFadden (1984). Both models have strong foundations in evolutionary and discrete choice modeling. This paper develops the dynamic environment and explores computationally the variety of processes and outcomes as determined by different combinations of the EWA and nested logit model parameters. An individual's characteristics can inform local experiences but aggregate outcomes are the product of the environment.

\section{Model}

Let $N=\{1, \ldots, n\}$ be a set of agents. Let $O(t)=\left\{O_{1 t}, O_{2 t}, \ldots, O_{m t}\right\}$ be a new set of options available in time period $t$. In each time period, $t \in\{1, \ldots, T\}$, each agent looks to adopt from one of the options. Let $K=\{$ " $A$ ", " $B$ ", $\ldots\}$ be a set of $m$ labels for these options and let the private one-to-one function $f_{i, t}$, determined by nature, map from labels to options, $f_{i, t}: K \rightarrow O(t)$. Each player thus privately observes the $K$ set of labeled options. Each player sees different labels and for every $i, j$ pair there is a one-to-one 
function $h_{i, j, t}: K_{i, t} \rightarrow K_{j, t}$ that is unknown to the players. The mechanism prevents coordination between agents on a particular choice, either through prior communication, or by identifying a focal point.

Visibility across the population is limited to individual contact lists which identify, for each agent, who in the population they can directly observe. Thus, agent $i$ can observe agent $j$ 's adoption of $O(t)$ only if agent $j$ is on $i$ 's contact list. Let $N^{d}(i) \subseteq N$ represent $i$ 's contact list. Let the adjacency matrix $g$ capture the network of contact links where element $g_{i j}=1$ if $j \in N^{d}(i)$ and zero otherwise. Let $n_{d}$ indicate the number of contacts for each agent, $n_{d}=\left|N^{d}(i)\right|$.

Let $a_{i, t}$ denote the action of player $i$ in period $t$. Players act simultaneously with each player choosing (i) one of the $m$ options, or (ii) to link to another player. In the former case, if player $i$ chooses option $O_{k, t}$, then assign $a_{i, t}=f_{i, t}^{-1}\left(O_{k, t}\right)$. If player $i$ links to player $j$, then assign $a_{i, t}=j$. A player who chooses an option is said to lead while a player who links to another is said to imitate or follow the other player. An agent who leads and has followers is referred to as a leader. Thus, the set of actions for player $i$ is $A_{i}=K_{i} \cup N^{d}(i)$. Write $a=\left(a_{1}, \ldots, a_{n}\right)$, for an action profile, where $a_{i} \in A_{i}$.

Each $a_{i} \in N^{d}(i)$ represents a decision to imitate the targeted individual. Let $\sigma$ represent the adjacency matrix produced by imitative actions so that $\sigma_{i j}=1$ if $a_{i}=j$ and zero otherwise. Refer to $\sigma$ as the social structure for capturing who leads and who follows whom. Let $\Sigma$ represent the set of $\sigma$ in which there is a single leader and the remaining $n-1$ agents follow the single leader, directly or indirectly through a series of links. Let $\Sigma^{*} \subseteq \Sigma$ be the set of structures in which each follower is at the minimum distance possible from the leader measured in links according to the available contacts.

Example 1. The two frames of Figure 1 present two possible realizations generated from the same set of underlying actions. The population consists of $n=7$ agents facing $m=3$ options. The action set is $a=(" A ", 1,1,3, " C ", 7,6)$. That is, both agents 1 and 5 lead, agents 2 and 3 imitate agent 1 , agent 4 imitates agent 3, agents 6 and 7 imitate each other. These actions determine the social structure for the period. Only agent 1 is a leader, having a non-trivial population of followers. Agents 2,3, and 4 are followers of 1 . Agent 5 leads, but is not a leader. Agents 6 and 7 are within a self-referencing loop.

What distinguishes the two realizations is the mapping from labels to options. In 1a, agents 1 and 5 select different options according to nature's determination of $O_{1}=f_{1}(A)$ and $O_{3}=f_{5}(C)$. In $1 \mathrm{~b}$, agents 1 and 5 select the same option according to nature's determination of $O_{2}=f_{1}(A)=f_{5}(C)$.

Allow that $i$ is a predecessor of $j$ and $j$ is a successor of $i$ in the structure $\sigma$ if $j$ imitates $i$, either directly or indirectly through a chain of imitators. Let $d_{i, j, t}$ represent the distance from agent $i$ to agent $j$ measured in the number of links that separate them. From Example $1, d_{2,1, t}=d_{3,1, t}=1$ and $d_{4,1, t}=2$. An agent's 

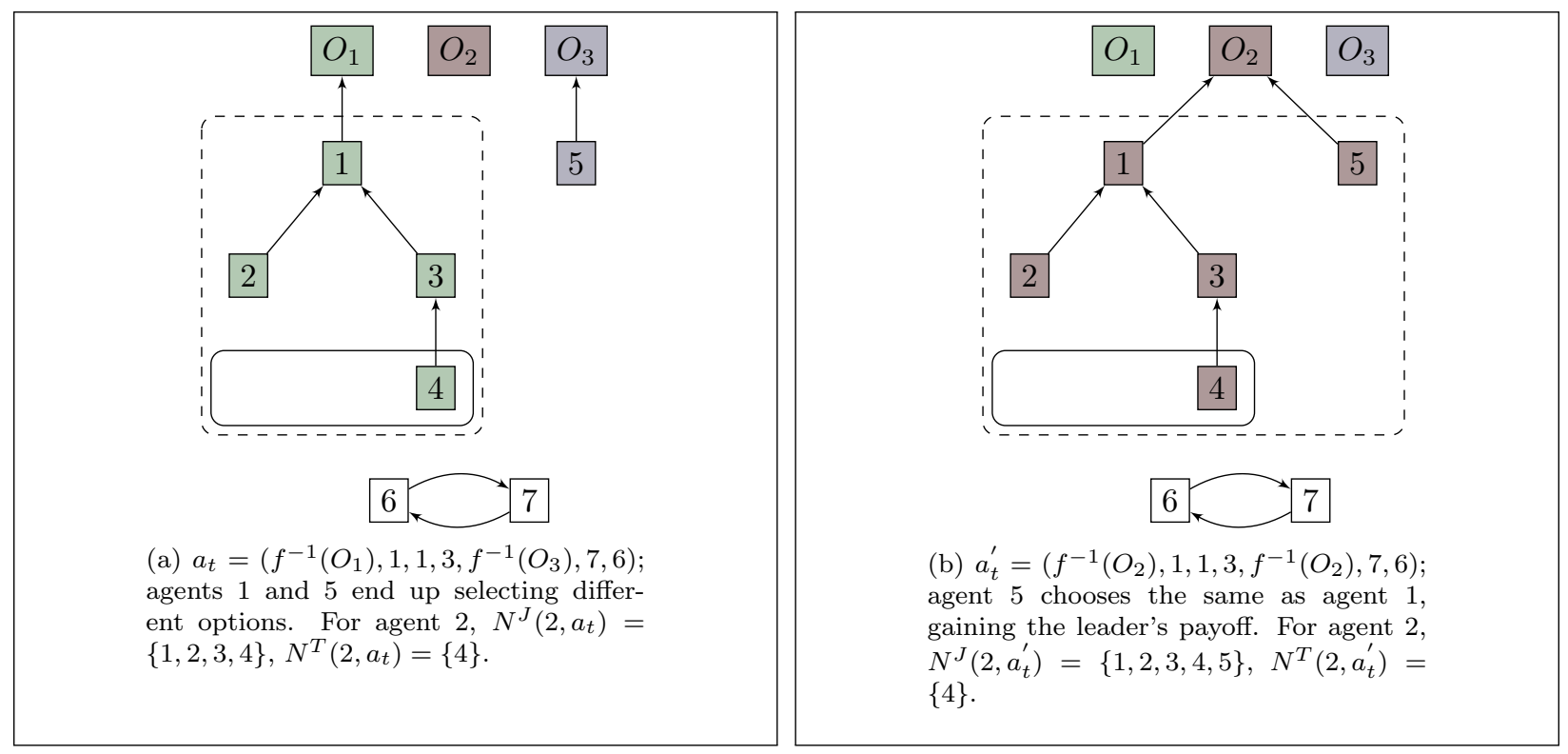

Figure 1: Choice and social organization in a population with $n=5, m=3$. Payoffs depend on an agent's relative distance from the option selected. An arrow from an individual (identified with a number) directly to one of the choices along the top of the frame (identified $O_{n}$ ) represents a choice by a leader. An arrow from one agent to another represents a path of imitation with the arrow originating from the imitator.

distance from the option she adopts can likewise be measured. Let $d_{i, t}$ represent the distance from agent $i$ to the option she adopts. Starting from a distance of zero for leaders, $d_{1, t}=d_{5, t}=0, d_{2, t}=d_{3, t}=1, d_{4, t}=2$, and $d_{6, t}=d_{7, t}=+\infty$.

The depiction in Figure 1 places the options at the top of the frame. Those who lead are directly below the options and those who follow are positioned vertically according to $d_{i, t}$. The depiction suggests a social hierarchy based on distance. A hierarchy is a tree structure with the leader as the root node and branches of successors. A tier applies to a set of successors at a shared distance. Time-to-adoption and distance from a leader become synonymous when each link imposes a delay between adoption by the target and the observation and subsequent adoption by the link's originator.

Let $N^{J}(i ; a)$ denote the set of players who adopt the same option as does player $i$ (exclusive of $i$ ). Let $N^{T}(i ; a)$ denote the subset of $N^{J}(i ; a)$ which is of greater distance from the option than is $i$. As illustration, the dashed box included in both frames of Figure 1 captures $N^{J}\left(2, a_{t}\right)$. The solid box captures $N^{T}\left(2, a_{t}\right)$. The members of $N^{T}(i ; a)$ need not be successors of $i$, just as agent 4 is not a successor of agent 2 but us a member of $N^{T}\left(2, a_{t}\right)$. 
Let $n_{i, t}^{J}$ and $n_{i, t}^{T}$ represent the size of the respective $N^{J}(i ; a)$ and $N^{T}(i ; a)$ sets. The payoff for player $i$ is

$$
\pi_{i}(\sigma)=a_{J} n_{i, t}^{J}+a_{T} n_{i, t}^{T}
$$

with coefficients $a_{T} \geq 0$ and $a_{J} \geq 0$. The first element of the payoff is the "conformity" component, much like the community effect of Blume and Durlauf (2001). The second element in (1) reflects the distance/timing advantage a player has over other players. By (1), an individual's payoff is purely a social phenomenon. The options themselves offer no direct benefit to the agent.

Autarky, the consequence of everyone leading, generates expected payoffs of $a_{J}(n-1) / m$. The full coordination produced by the entire population formed into a single hierarchy generates individual payoffs ranging from $\left(a_{T}+a_{J}\right)(n-1)$ for the leader to $a_{J}(n-1)$ going to the most distant follower(s). The benefit over autarky to even the lowest compensated agent illustrates the benefit to coordinating.

Let

$$
B:=\theta-\left(1-\frac{1}{n-1}\right)
$$

with $\theta=(m-1) a_{J} / a_{T}$. The parameter $\theta$ reflect the relatives strength between the attraction for conformity and the attraction to lead. The most distant successors are attracted to lead when the premium to acting in advance of followers is high (so that $a_{J} / a_{T}$ is low) or when $m$ is small, yielding a high probability of choosing the same as the leader.

The set of equilibrium structures always includes a single hierarchy structure. The equilibrium size of the hierarchy increases with $B$ with $B \geq 0$ inducing the entire population to join in following the single leader. Abandonment of the hierarchy by followers is from the bottom as an already negative $B$ decreases. The equilibrium hierarchy size for $B<0$ has a number of followers, $n_{s}^{*}<n-1$ with $n_{s}^{*}$ an integer value near $1 /(1-\theta)$.

If the equilibrium structure is going to emerge in the simultaneous decision setting, then it has to emerge from path dependent play. Simulations are employed to investigate dynamic behavior and adjustment to explore possible paths of individual behavior and group organization.

For the dynamic simulation, each agent chooses from the available actions probabilistically. In period $t$, for $\rho_{i, t} \in[0,1]$,

$$
\begin{aligned}
\rho_{i, t} & =\operatorname{Pr}(i \text { leads }) \\
\left(1-\rho_{i, t}\right) w_{i, t}^{j} & =\operatorname{Pr}(i \text { follows } j) j \in N^{d}(i),
\end{aligned}
$$


where $\sum_{j \in N^{d}(i)} w_{i, t}^{j}=1, w_{i, t}^{j} \in[0,1] \forall i, j$. For those agents who lead, a simple random assignment for determining $k_{i, t} \in O_{t}$ captures the inability to coordinate on an initial choice.

Individual performance associated with each strategy option is tracked and updated according to the experience weighted attractor (EWA) of Camerer and Ho (1999). That is, agent $i$ maintains a performance measure associated with leading,

$$
\begin{aligned}
A_{0, t+1}(i) & =\frac{\phi_{l} N_{t, l} A_{0, t}+\left(\delta_{l}+\left(1-\delta_{l}\right) \mathbf{1}\left(x_{t}=l\right)\right) \pi_{l, t}}{N_{t+1, l}} \\
N_{t+1, l} & =1+\phi_{l}\left(1-\kappa_{l}\right) N_{t, l}
\end{aligned}
$$

and for following each agent $j \in N^{d}(i)$,

$$
\begin{aligned}
A_{j, t+1}(i) & =\frac{\phi_{f} N_{t, f} A_{j, t}+\left(\delta_{f}+\left(1-\delta_{f}\right) \mathbf{1}\left(x_{t}=d\right)\right) \pi_{j, t}}{N_{t+1, f}} \\
N_{t+1, f} & =1+\phi_{f}\left(1-\kappa_{f}\right) N_{t, f} .
\end{aligned}
$$

Relative performance determines the probability of adoption of an action according to the nested logit model. For performance measures $A_{0}, A_{1}, \ldots, A_{d}$,

$$
\begin{gathered}
\operatorname{Pr}(i \text { leads })=\rho_{i, t}=\frac{\exp \left(\mu A_{0, t}\right)}{\exp \left(\mu A_{0, t}\right)+\left(\sum_{j \in D} \exp \left(\mu A_{j, t} / \lambda\right)\right)^{\lambda}} \\
\operatorname{Pr}(i \text { follows } j)=\left(1-\rho_{i, t}\right) w_{i, t}^{j}=\frac{\exp \left(\mu A_{j, t} / \lambda\right)\left(\sum_{j \in D} \exp \left(\mu A_{j, t} / \lambda\right)\right)^{\lambda-1}}{\exp \left(\mu A_{0, t}\right)+\left(\sum_{j \in D} \exp \left(\mu A_{j, t} / \lambda\right)\right)^{\lambda}} .
\end{gathered}
$$

As shaped by the parameters of the EWA, the performance of each strategy is updated based on the most recent realized payoff or counterfactual. Decreasing $\phi$ from one towards zero shifts weight towards more recent performance realizations, producing "recency" in the weighting of past performance. Decreasing $\delta$ from one towards zero down-weights the counterfactual payoffs so that at the extreme $\delta=0$ the agent engages in reinforcement learning. For $\kappa \rightarrow 1$, persistent performance differences accumulate over time to drive probabilities towards the extremes, known as "lock-in".

In empirical studies, a nested logit is required when there is correlation between the choices in a multinomial logit environment. The generalized extreme value distribution upon which the nested logit is based allows for correlation within the "nested" options and is useful for decomposing choice options that are separated by an implicit sequential ordering of decisions in a tree structure or by some other grouping mechanism. 
In a simulation setting the nesting serves as a mechanism for compartmentalizing components of a decision.

The parameter $\mu$ is commonly referred to as the intensity of choice (IOC) parameter. It determines how sensitive an agent is to differences in the performance measure. At $\mu=0$, the agent is indifferent to the choices regardless of the performance. As $\mu$ increases, for a fixed performance differential, the agent's probability of adopting the superior strategy increases.

The $\lambda$ parameter controls the extent to which the agent compartmentalizes the decision about whether to lead or follow from the decision about who to imitate when following. Nesting addresses the independence of irrelevant alternatives problem in multinomial logit problems. The nested logit allows for the introduction of a new contact to draw probability weight from the other contacts in different proportion than it draws from the option to lead.

The advantage of the EWA and nested logit combination is that the models are well grounded empirically and have been used extensively for estimating human behavior in a wide range of discrete choice settings. The model performs poorly when examined using a discrete choice model without nesting because it forces a single IOC parameter to capture the intensity of both the decision about whether to lead or follow and the decision about who to follow. Completely separating the decision about whether to follow from the decision about who to follow using a two step decision process lacks this empirical foundation and, in particular, fails to preserve independence from irrelevant alternatives. ${ }^{4}$

Once actions have been determined for the period, the action-dependent social network structure $\sigma$ can be constructed, option selection by leaders made, and payoffs computed according to (1). The EWA updates the performance measures of the untried options as well as the options employed. For the former, the agents need to estimate the payoff that would have been earned with each of the counterfactual actions. Ex-post, the agents can observe which option each member of their contact list adopted and when, within the period, this adoption took place. With knowledge about aggregate popularity of each option as it disseminates through the population, the agents can place themselves in the position of having imitated each contact and guess at the position dependent counterfactual payoff earned, treating the time of adoption for all others as given.

\footnotetext{
${ }^{4} \mathrm{~A}$ variety of alternatives to the combined EWA and nested logit are available for dynamically modeling strategy adjustment. Also modeled, but not included for presentation in this paper, were two versions of a two-stage decision process with separate EWA processes for determining the value of $\rho_{i, t}$ and for determining the value of $w_{i, t}^{j}$. The two-stage decision was investigated using both the Camerer and Ho (1999) specified power distribution for allocating probabilities based on the performance measures as well as an alternate method based on a $k$-choice replicator dynamics derived from Branch and McGough (2008). The latter is considerably more resilient at achieving the equilibrium structure from a variety of starting conditions and environment setting than is the EWA and nested logit combination.
} 


\section{Simulations}

\subsection{Measuring organizational success}

One measure of how close the population has come to adopting equilibrium strategies is to look at the number of agents who lead. Let $\mu_{\text {lead, } t}$ be the number of agents who lead in period $t$. For $B \geq 0, \mu_{\text {lead, } t}=1$ in equilibrium. Another measure of success is to consider each agent's distance from their chosen leader. A minimal distance social structure is one in which each follower chooses to rely on the contact offering the shortest distance to her leader.

Recall that $d_{j, i, t}$ represents agent $j$ 's distance measured in number of links from leader $i$ in period $t$, let $\underline{d}_{j, i, t}$ indicate the shortest possible distance available to player $j$ given the linking decisions of the other agents. Let $\Delta_{t}$, referred to as the $\Delta$-inefficiency score, measure aggregate deviations from minimal distance according to

$$
\Delta_{t}=\sum_{j} d_{j, i, t}-\underline{d}_{j, i, t}
$$

Accordingly, $\Delta_{t}=0$ represents a social structure in which each follower employs the shortest distance to her leader. Values of $\Delta_{t}>0$ indicate deviations from minimal distance. A third related measure of the proximity to the equilibrium structure is how many in the population are caught in a self-referencing imitation loop. Let $\mu_{\text {loop }, t}$ represent the number of agents caught in a self-referencing loop.

The variation in the parameters across different treatments leaves $B$ unchanged, and thus only affects the evolutionary process of the population and not the equilibrium target.

\subsection{Behavior effects}

This section presents simulations of a relatively large homogenous population distributed over a randomly generated, strongly connected, directed network capturing individual contact lists. Table 1 reports the model treatments considered.

Frequencies with which each treatment achieves or nearly achieves the equilibrium structure are derived from Monte Carlo simulations. An illustrative example of each treatment is presented and discussed qualitatively.

For $B \gg 0$, there is a strong incentive to seek conformity and comparatively weak incentive to lead. In such a reward setting, simulations quickly achieve the cooperative structure for an expansive combination of the adaptive parameters. More interesting is a $B$ that is just above or any $B<0$. At certain positions in 
Table 1: Simulation Treatments

\begin{tabular}{|c|c|c|c|c|c|c|c|c|c|c|c|c|c|}
\hline Treatment & & Base & 1 & 2 & 3 & 4 & 5 & 6 & 7 & 8 & 9 & 10 & 11 \\
\hline Population & $n$ & \multicolumn{12}{|c|}{100} \\
\hline Number of contacts & $n_{d}$ & \multicolumn{12}{|c|}{6} \\
\hline Number of options & $m$ & \multicolumn{12}{|c|}{12} \\
\hline Periods & $T$ & 500 & 500 & 500 & 500 & 1000 & 500 & 500 & 500 & 500 & 500 & 500 & 500 \\
\hline Conformity reward & $a_{J}$ & 0.2 & 0.1 & 0.1 & 0.1 & 0.1 & 0.1 & 0.1 & 0.1 & 0.1 & 0.1 & 0.1 & 0.1 \\
\hline Preemption reward & $a_{T}$ & & & & & & 1 & & & & & & \\
\hline Participation & $B$ & 1.21 & 0.11 & 0.11 & 0.11 & 0.11 & 0.11 & 0.11 & 0.11 & 0.11 & 0.11 & -0.06 & -0.06 \\
\hline Eq. Hierarchy size & $n_{s}^{*}$ & 100 & 100 & 100 & 100 & 100 & 100 & 100 & 100 & 100 & 100 & 15 & 15 \\
\hline Recency (L) & $\phi_{l}$ & 0.9 & 0.9 & 0.9 & 0.9 & 0.99 & 0 & 0.9 & 0.9 & 0.9 & 0.9 & 0.9 & 0.99 \\
\hline Recency $(\mathrm{F})$ & $\phi_{f}$ & 0.9 & 0.9 & 0.9 & 0.9 & 0.99 & 0 & 0.9 & 0.9 & 0.9 & 0.9 & 0.9 & 0.99 \\
\hline Lock-in (L) & $\kappa_{l}$ & 0 & 0 & 0 & 0 & 0.2 & 0 & 0 & 0 & 0 & 0 & 0 & 0.2 \\
\hline Lock-in (F) & $\kappa_{f}$ & 0 & 0 & 0 & 0 & 0.2 & 0 & 0 & 0 & 0 & 0 & 0 & 0.2 \\
\hline Reinforcement (L) & $\delta_{l}$ & 1 & 1 & 1 & 1 & 1 & 1 & 0.2 & 1 & 1 & 1 & 1 & 1 \\
\hline Reinforcement (F) & $\delta_{f}$ & 1 & 1 & 1 & 1 & 1 & 1 & 1 & 0.7 & 1 & 1 & 1 & 1 \\
\hline Prior on leading & $A_{0}(0)$ & 0 & 0 & 0 & 0 & 0 & 0 & 0 & 0 & 0 & 3.5 & 0 & 0 \\
\hline Intensity of choice & $\mu$ & 1 & 1 & 3 & 0.05 & 0.2 & 0.6 & 1 & 1 & 1 & 1 & 0.6 & 0.2 \\
\hline Independence & $\lambda$ & 0.9 & 0.9 & 0.9 & 0.9 & 0.9 & 0.9 & 0.9 & 0.9 & 0.15 & 0.9 & 0.9 & 0.9 \\
\hline \multicolumn{2}{|l|}{ iterations } & 200 & 200 & 200 & 200 & 200 & 200 & 200 & 200 & 200 & 200 & 200 & 200 \\
\hline \multicolumn{2}{|l|}{ Freq. $n_{s}=99$} & 0.995 & 0.470 & 0.705 & 0 & 0.980 & 0.305 & 0.995 & 0 & 0 & 0.400 & 0.025 & 0 \\
\hline \multicolumn{2}{|l|}{ Freq. $n_{s} \geq 95$} & 1 & 0.810 & 0.770 & 0 & 0.995 & 0.575 & 1 & 0 & 0 & 0.840 & 0.335 & 0.005 \\
\hline \multicolumn{2}{|l|}{ Freq. $n_{s} \geq 50$} & 1 & 0.990 & 0.805 & 0.120 & 0.995 & 0.965 & 1 & 0.485 & 0.48 & 0.985 & 1 & 0.470 \\
\hline
\end{tabular}

the social structure, the differential by which between following and leading is small. How individuals and those around them process information can impact imitation network evolution and outcomes.

A wide range of behaviors produce regular emergence of a near-equilibrium social structures. Consistently producing the exact equilibrium structure requires a narrower set of parameters, particularly as $B \rightarrow 0$ from above and for $B<0$.

The ex ante homogeneity causes players to employ identical first-period probabilities in identifying an action. The different personal experiences in the first and subsequent periods lead to individual updating of probabilities and thus ex post heterogeneity. Through this process, transitive events have potentially permanent consequences by becoming embedded in the social network. For a leader to emerge, others in the population must adjust $\rho_{i}$ and $w_{i, t}^{j} \forall j \in N^{d}(i)$ to chase success. The process of observation and adjustment allows the initially lucky individual to become a successful leader. The established leader is ensured of a good outcome in each decision round, no longer reliant on luck but empowered by her followers. The success in generating the equilibrium structure is in its backwards-looking measure of performance and the induced adjustments in strategy that reward success with more success.

Figure 2 displays the typical first period structure under three different scenarios capturing the impact 


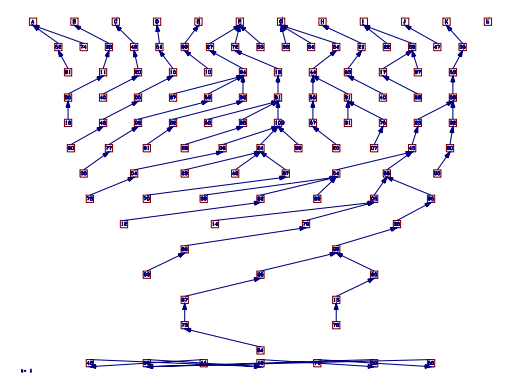

(a) No nesting, no bias: $\lambda=1, A_{0,0}=0$

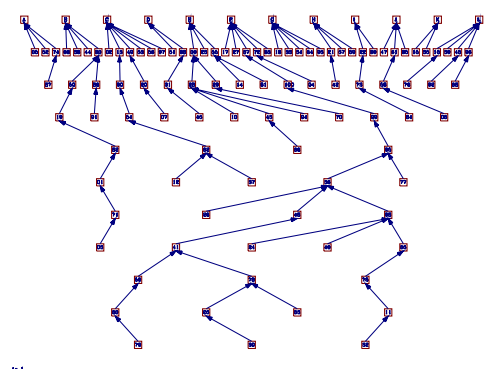

(b) Nesting: $\lambda=0.1, A_{0,0}=0$

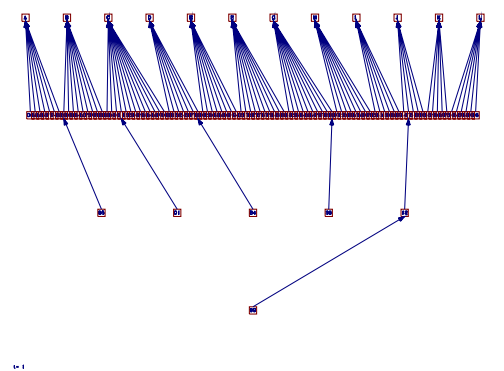

(c) Bias: $\lambda=1, A_{0,0}=5$

Figure 2: Initial structure at $t=1$ : $(2 \mathrm{a})$ With independence across decisions and no bias, $\rho_{i}=0.14 \forall i$. Long chains of followers with multiple branches produce considerable heterogeneity in $t=1$ individual's experiences that feed into $t=2$ decisions. (2b) With near-complete nesting, $\rho_{i}=0.46 \forall i$. Long chains are present but multiple agents choose the same options, obscuring to the decision makers which agent has the followers. (2c) With a preference to lead producing $\rho_{i}=0.96 \forall i$, there is minimal differentiation in payoffs.

of nesting and of a predisposition to leading

\subsubsection{Baseline}

The important feature of the baseline treatment is the relatively strong preference for conformity. The $B=$ 1.2 is sufficiently large so that following stands out as an attractive option supported by prior experiences. Once the hierarchy is formed with $\sigma \in \Sigma$, the most distant follower observes from personal experience that the certain conformity that comes from following offers greater reward than the expected value of leading.

With the strong incentive for conformity, the process of emergence to the coordinating structure is robust to a wide range of possible behavioral model parameters. For illustrative purposes, the EWA parameters employed in this treatment are chosen to unbiasedly reflect underlying differences in performance. The agents employ long memory and give full weight to counterfactual payoffs of the untried actions, but these features are not essential.

The frames of Figure 3 present information capturing the evolution and eventual outcome from a chosen revealing simulation. The sparse 3 a reveals the speed with which the population joins in following a single leader so that the three measures of $\mu_{\text {lead }}, \mu_{\text {loop }}$, and $\Delta$ all quickly drop to zero. Frame $3 \mathrm{~b}$ shows the social structure as realized in the terminal period. Frames 3c-3f present the time-series of the performance measures for the leader and select individuals based on their final distance from the leader. The overwhelming desire for conformity ensures that for even the most distant follower, following clearly dominates leading. The highly differentiated payoffs in the first period, as seen in Figure 2a, facilitate quick identification of a single leader and evolution to the equilibrium structure. 


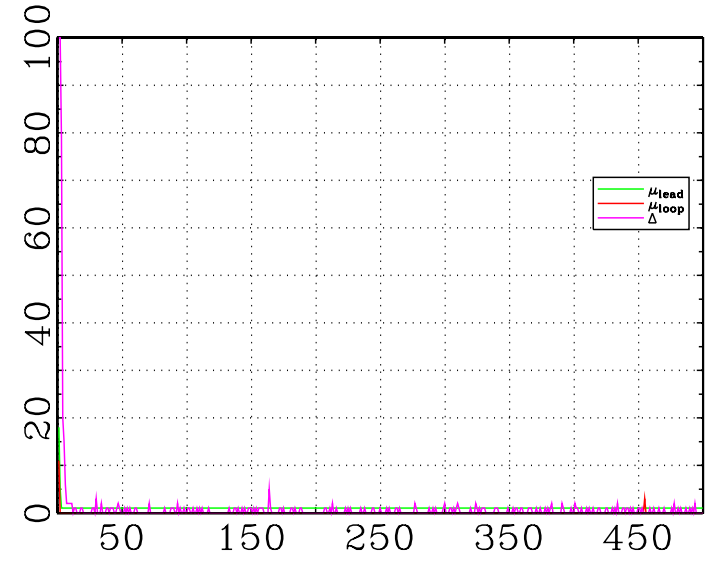

(a) Time-series of $\left\{\mu_{\text {lead }}, \mu_{\text {loop }}, \Delta\right\}=\{$ number lead, number looped, inefficiency $\}$.

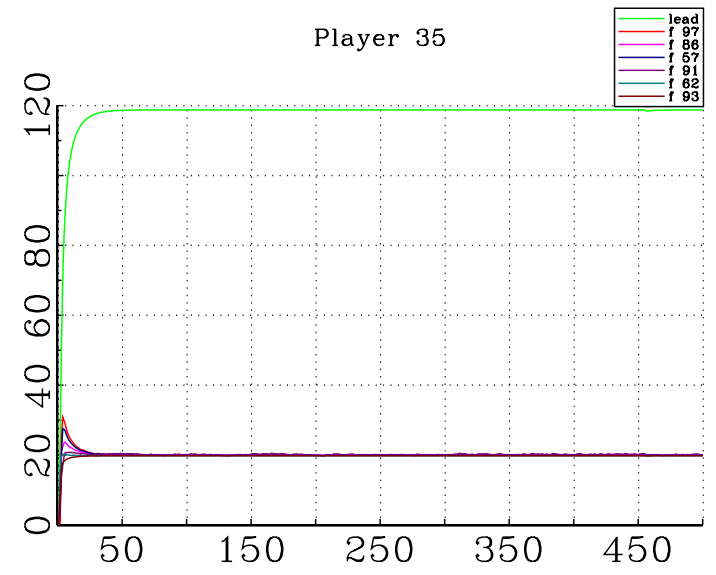

(c) Time-series $A_{0}-A_{6}$ for the eventual leader.

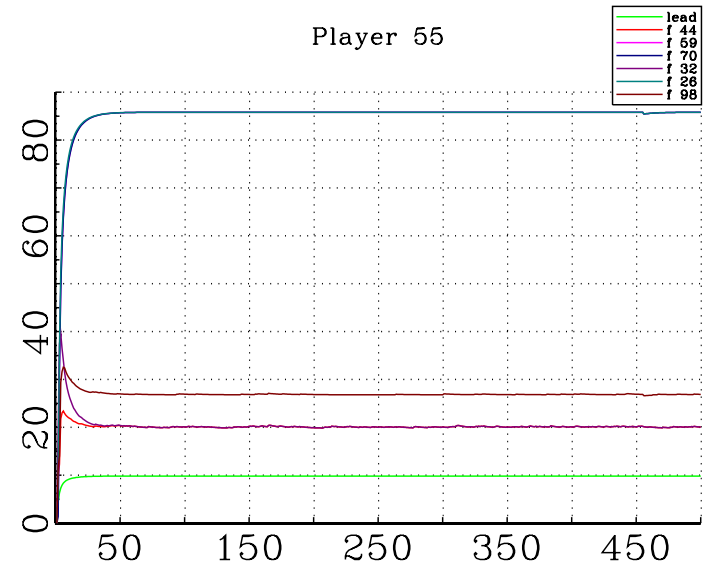

(e) Time-series $A_{0}-A_{6}$ for an agent two links from the leader.

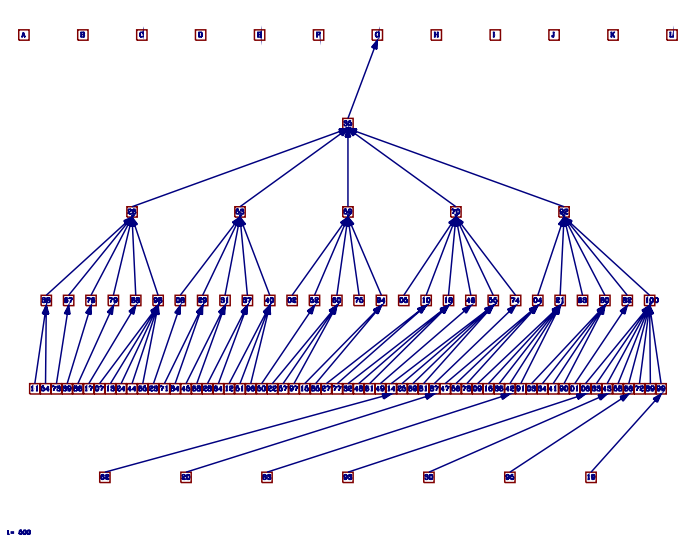

(b) Social structure in the final period $(T=500)$. A full participation $\Delta$-efficient hierarchy.

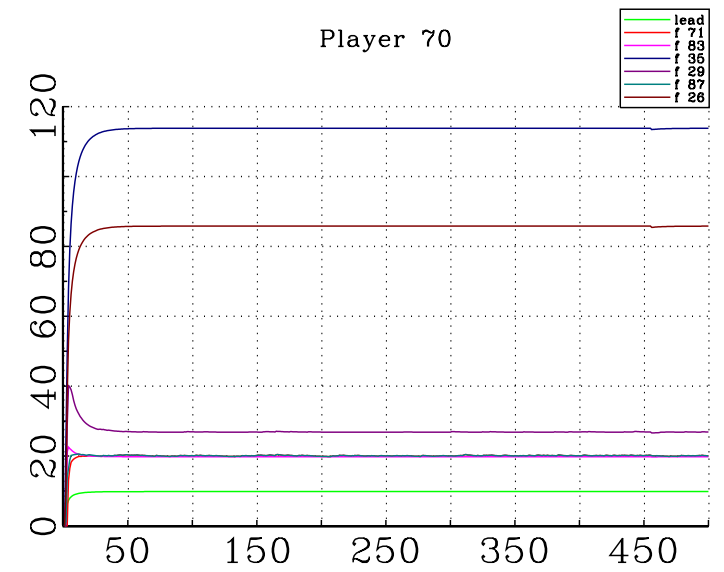

(d) Time-series $A_{0}-A_{6}$ for an agent one link from the leader.

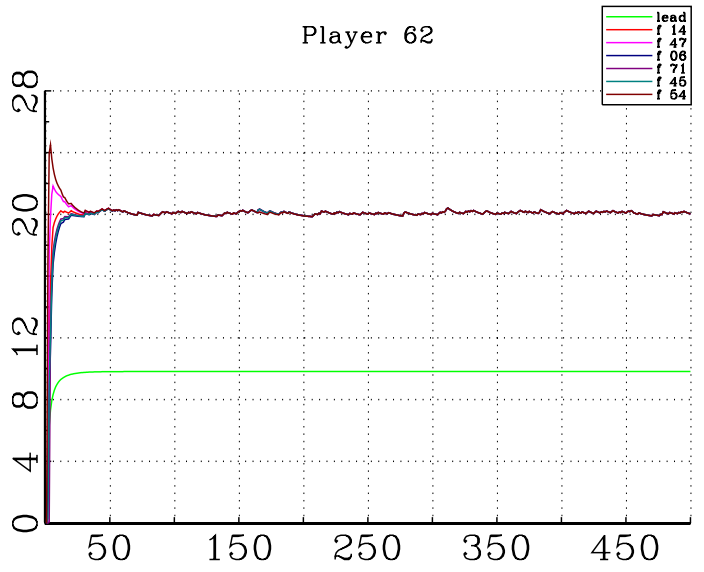

(f) Time-series $A_{0}-A_{6}$ for an agent most distant from the leader.

Figure 3: Baseline: $B=1.21$ The strong reward to conformity ensures the near immediate emergence of the coordinating equilibrium structure and no disruptions. 
The one instance out of 200 iterations, in which $n_{s} \neq n-1$ consists of two followers were caught in a self-referencing loop, following each other in an attempt to link indirectly to the leader. There were no instances where an agent other than the leader led in the final period.

\subsubsection{Conformity vs influence}

In Treatment 1, lowering $a_{J}$ decreases $B$ to 0.11 . As long as $B>0$, the target Nash equilibrium structure is unchanged but for $B$ close to zero, the payoff differential narrows, hindering identification of the superior action. Narrow performance differentials generate indecisiveness in a logit model with the inferior action garnering non-zero probability. This can potentially hinder evolution towards the equilibrium structure. Even if a leader is identified, the most distant followers, for whom following only narrowly dominates leading, are likely to remain irregular followers.

The most distant follower experiences one of three events each period. These are evident in the time-series of performance measures presented in Figure 4f. Following is the most common action, earning a payoff of $a_{J} n_{s}$. To lead and match the leader, earning a payoff of $a_{J} n_{s}+a_{T}\left(n_{s}-1\right)$, produces a spike up in $A_{0}$. To lead and fail to match the leader, earning a payoff of zero, produces a dip in $A_{0}$.

Presented in Figure 4 is one of only two instances in which the structure deviated considerably from the equilibrium. Here, the simulation is allowed to run for 800 periods to let the major disruption occurring around $t=475$ to play out. Though the disruption lasts for 200 periods, the population does return to the near-equilibrium structure. In the other instance (not graphed), the emergent dominant leader has only three direct followers, less than the average of six. From the onset, the followers remain in a seemingly stable structure in which there remains a persistent population of 20-40 who lead rather than follow. At about $t=700$, the population quickly transitions to form into a near-equilibrium structure.

\subsubsection{Intensity of choice}

Treatment 2 is based on an increase to the IOC parameter, $\mu$. The treatment is a possible counter to the diminished performance differential between leading and following observed in Treatment 1 . Increasing $\mu$, though, does not return the system to rapid convergence on the equilibrium structure. Compared to Treatment 1, increasing the IOC parameter increases the likelihood of achieving the equilibrium structure. It also increases the frequency with which little or no social structure emerges. Among the $20 \%$ of the runs that fail to produce at least 50 followers in the dominant hierarchy, $5 \%$ terminate having settled into a revolving population of only 10-20 agents following each period. The other $15 \%$ of the runs settle into an 


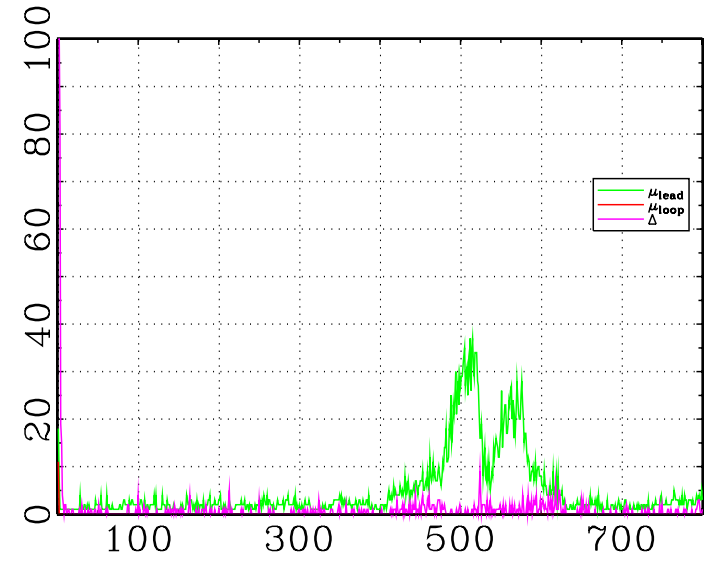

(a) Time-series of $\left\{\mu_{\text {lead }}, \mu_{\text {loop }}, \Delta\right\}=\{$ number lead, number looped, inefficiency $\}$.

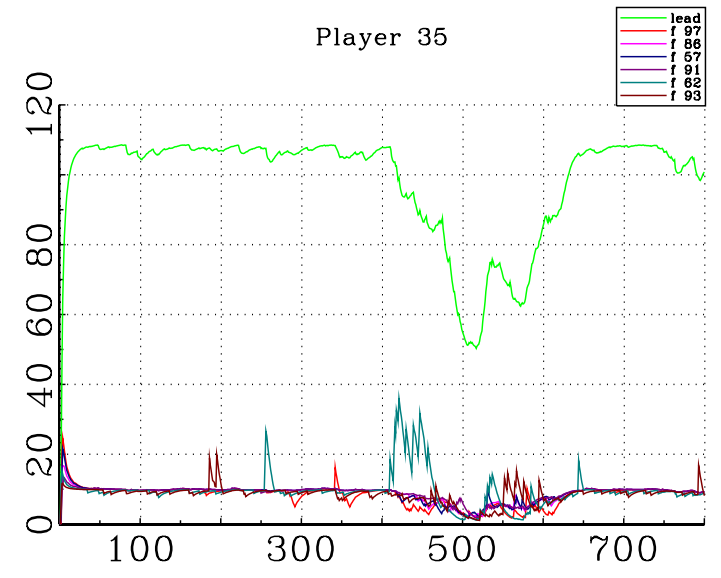

(c) Time-series $A_{0}-A_{6}$ for the eventual leader.

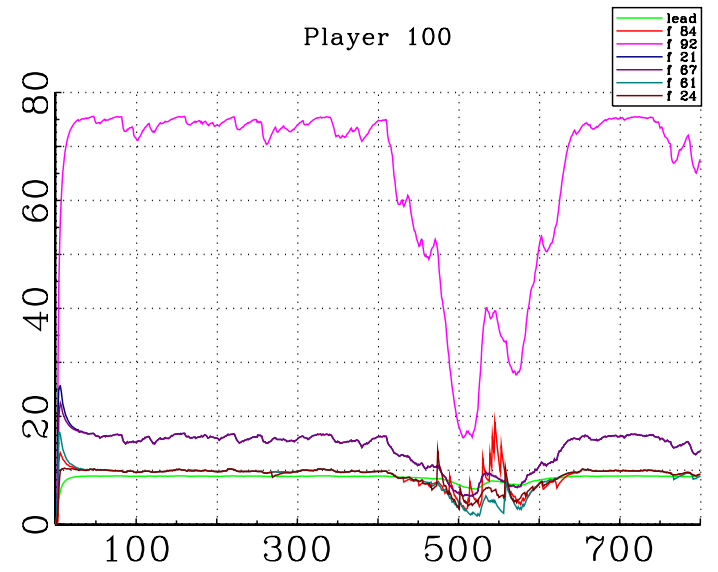

(e) Time-series $A_{0}-A_{6}$ for an agent two links from the leader.

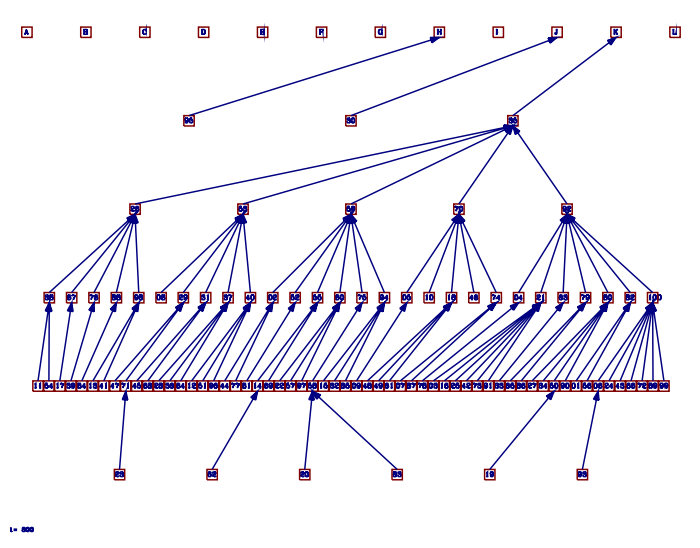

(b) Social structure in the final period $(T=800)$.

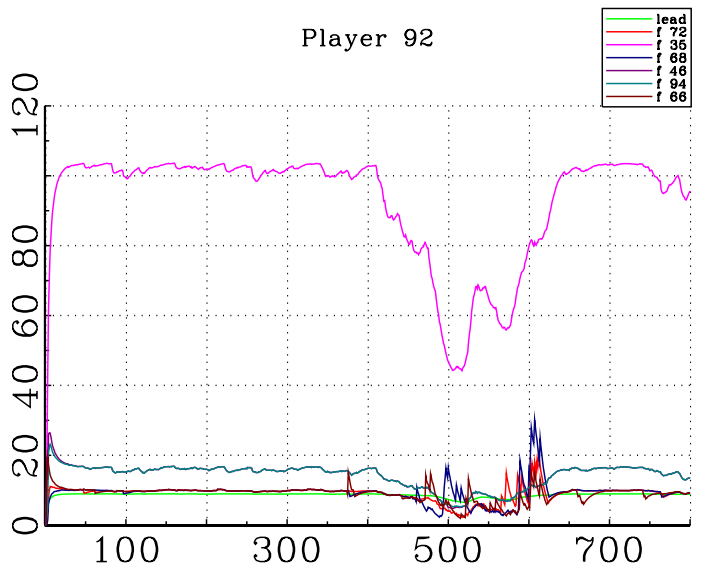

(d) Time-series $A_{0}-A_{6}$ for an agent one link from the leader.

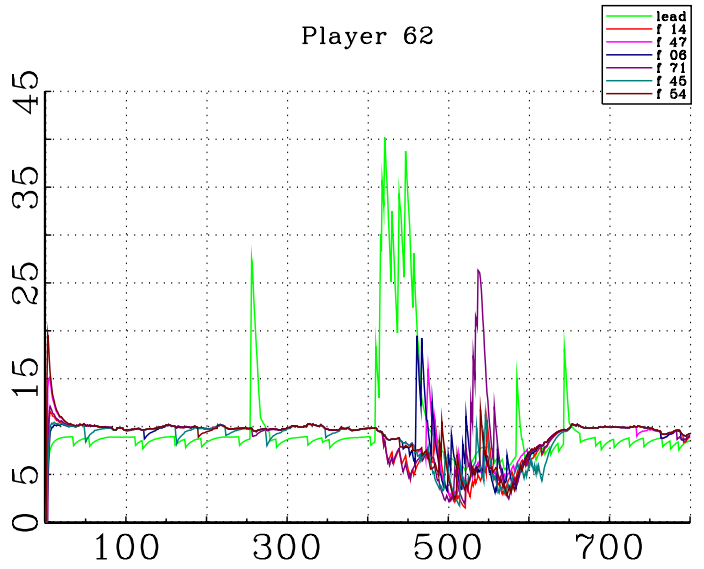

(f) Time-series $A_{0}-A_{6}$ for an agent most distant from the leader.

Figure 4: Small preference for conformity: With $B=0.11>0$ the reward to conformity remains stronger than the desire to lead but the small differential produces indecision among the most distant followers about whether to lead or follow, resulting in random adoption. 
apparently stable structure consisting of a single dominant leader but with only a fraction of the population following. Further increases in $\mu$ further increase the frequency in which no leader attracts a majority of the population as followers.

Extending the duration of the simulation provides the opportunity to achieve the more cooperative structure. Half of the runs with fewer than 50 followers at $t=500$ achieve the equilibrium structure when allowed to run 5,000 periods. One such run, presented in Figure 5 has no structure at time $t=500$. Around $t=1000$ the population shifts to a state in which a single leader leads a small but stable population of tier 1 followers. Around $t=4670$, there is another transition adding a second tier of followers to the hierarchy, quickly followed by a third. The transitions are quick, taking five periods or so each. The overall impression is that of a system transitioning through a series of locally stable disequilibrium structures before finally achieving an equilibrium cooperative structure. The possibility of a stable disequilibrium is developed in subsection 3.2.8.

Allowed to run for 10,000 periods, there remaining 11 runs that still fail to converged to the cooperative structure. All look to be in the locally stable disequilibrium consisting of a leader and single tier of followers.

Decreasing the IOC parameter for Treatment 3 decreases agent sensitivity to performance differentials. At a sufficiently low value, individual adjustment fails to respond to transitory random events sufficiently to create permanent social advantage. The result is a population that remains perpetually disorganized. The social structure in the last period is the same characteristically random structure as observed in the first, as seen in $2 \mathrm{a}$.

\subsubsection{Lock-in}

Treatment 4 introduces lock-in. The lock-in parameter adjusts how the agents aggregate performance information over time. Let $\pi_{h, t}^{\delta}$ reflect the performance of strategy $h=f, l$ in period $t$ after discounting counterfactual estimates according to $\delta_{h}$. Thus,

$$
\pi_{h, t}^{\delta}=\left(\delta_{h}+\left(1-\delta_{h}\right) \mathbf{1}\left(x_{t}=h\right)\right) \pi_{h, t}
$$

The impact of lock-in on the performance measure can be seen by examining the formula for the realized $A_{h, t+1}$, where, for $h \in\{0,1, \ldots, d\}$,

$$
A_{h, t}=\left(\sum_{s=0}^{t-1}\left(\phi_{h}\left(1-\kappa_{h}\right)\right)^{s}\right)^{-1}\left(\sum_{s=0}^{t-1}\left(\phi^{s} \pi_{h, t-s}^{\delta}\right)\right)
$$




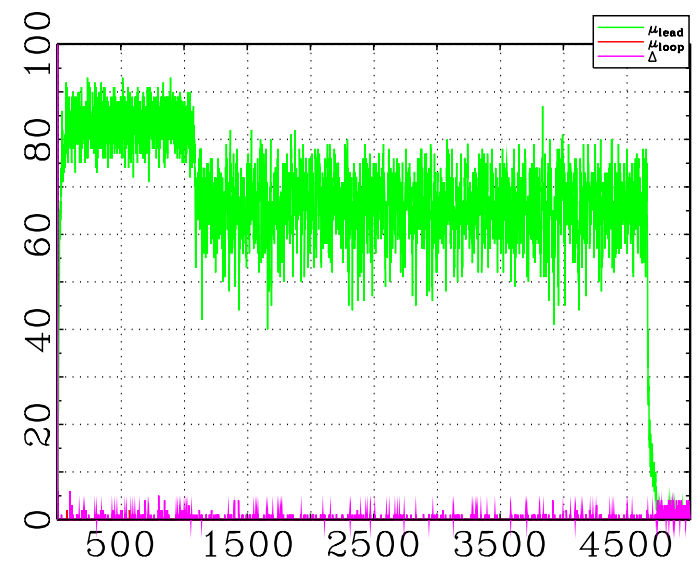

(a) Time-series of $\left\{\mu_{\text {lead }}, \mu_{\text {loop }}, \Delta\right\}=\{$ number lead, number looped, inefficiency $\}$.

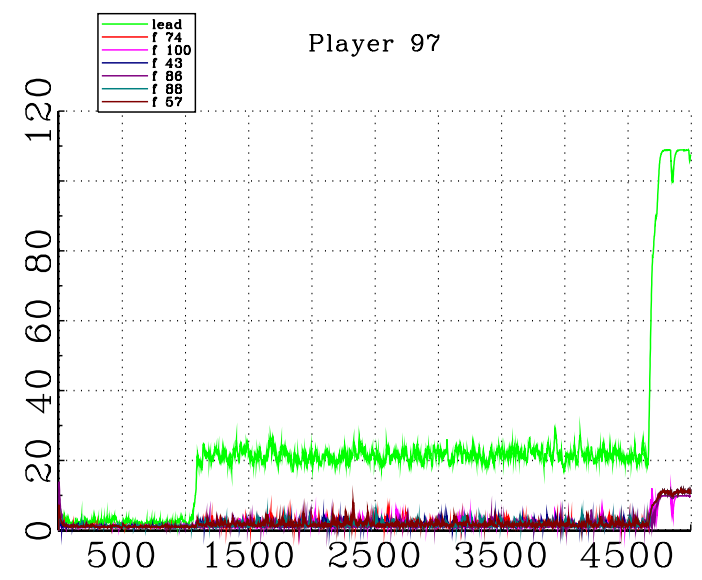

(c) Time-series $A_{0}-A_{6}$ for the eventual leader.

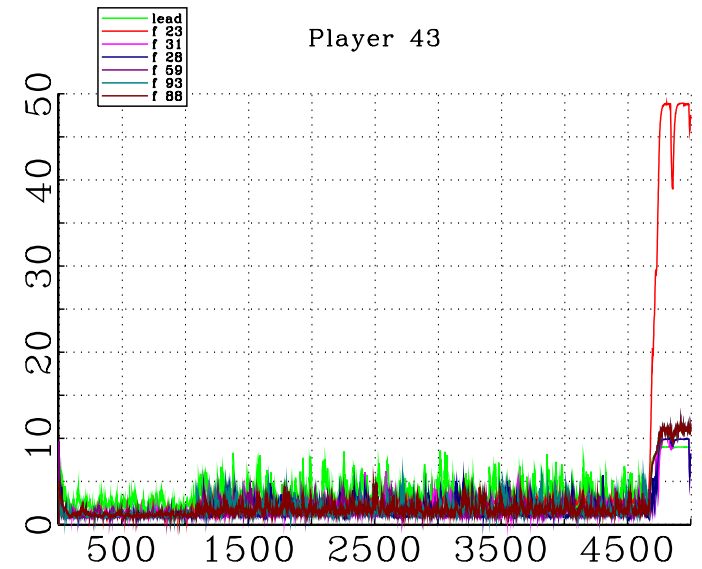

(e) Time-series $A_{0}-A_{6}$ for an agent two links from the leader.

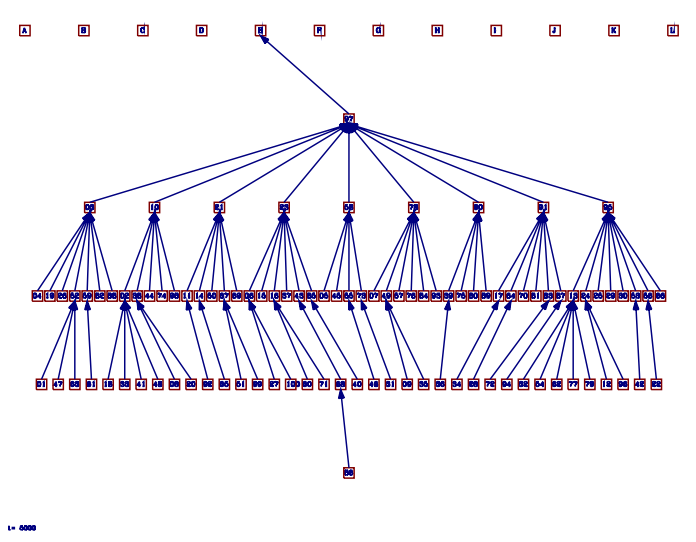

(b) Social structure in the final period $(T=5000)$. Each distance $d_{j, i}=3$ followers has a small probability of ending up at distance 4 but never lead.

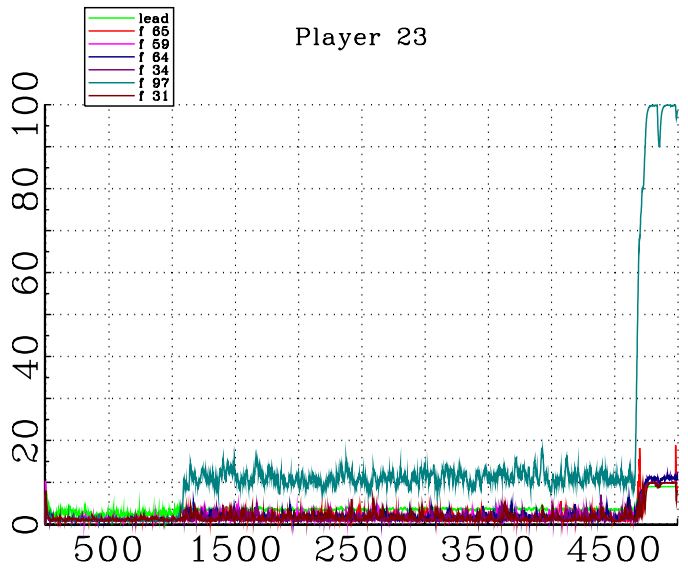

(d) Time-series $A_{0}-A_{6}$ for an agent one link from the leader.

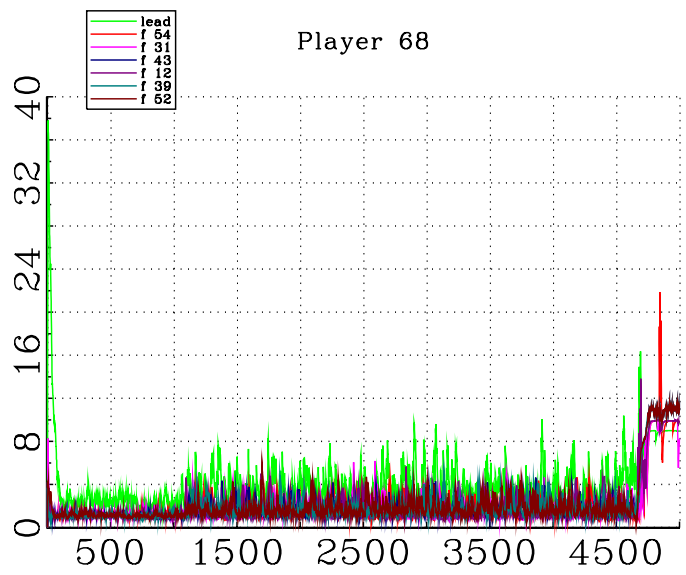

(f) Time-series $A_{0}-A_{6}$ for an agent most distant from the leader.

Figure 5: Increase IOC: $\uparrow \mu$ introduces instances in which the population appears to settle at a stable disequilibrium structure. 
For $\kappa_{h}=0, A_{h t}$ reflects the average of weighted past performances. For $\kappa_{h}=1, A_{h, t}$ reflects an accumulation of past performances. The distinction is that for $\kappa_{h}=0$, the expected performance differential is the average of the past performance differences, with the weighted sum of the past performances divided by the sum of the weights. For $\kappa_{h}=1$, the expected performance differential is the sum of the past differences. Accumulating past performance rather than averaging allows a persistent differential to accumulate greater performance on the superior option. The process could be interpreted as an increase in confidence through the accumulation of information that allows the agent to put greater probability on the superior performing action.

For a $\pi_{h, t}$ drawn from a stationary distribution with mean $\bar{\pi}_{h}$, then for $\phi<1, A_{h, t}$ converges to a stationary distribution around a fixed mean value,

$$
\bar{A}_{h}=\frac{1-\phi_{h}\left(1-\kappa_{h}\right)}{1-\phi_{h}} \bar{\pi}_{h}
$$

For a $\kappa \neq 0$ to meaningfully impact behavior requires the long memory produced by $\phi_{h}$ at or near one. The infinite memory implied by $\phi=1$ and $\kappa=1$ ensures that a consistently perceived dominating action eventually attracts all of the probability weight, even for finite $\mu$. From (8), $\kappa_{h}>\kappa_{-h}$ generates an asymptotic bias in favor of the $h$ associated action.

Finite machine measurability precludes simulating $\phi=1$ and $\kappa=1$. Thus, the treatment employs $\phi=0.992$ and $\kappa=0.2$. Two additional accommodations lower $\mu$ and increase the length of each run to $T=1000$. The longer run provides time for an accumulation of evidence while the lower $\mu$ keeps the nested logit computed probabilities machine measurable. This combination of features substantially raises the incidence of emergence of the equilibrium structure. Instances in which the system settles at one of the locally stable disequilibria still arise. The accumulation effect of employing lock-in can be seen in the rising trend of the performance measure in Figure 6.

\subsubsection{Recency}

Treatment 5 introduces recency by setting $\phi_{l}=\phi_{f}=0$. A leader emerges as behavior reinforces positive experiences. Individual memory is not essential in this process as the network grows to reflect individual experiences. Increasing recency by lowering $\phi$ leaves in place the ability to generate a dominant leader but introduces instability in the social structure. The non-zero probability to lead means that there is always deviation from the equilibrium structure. The absence of memory opens the possibility that the randomness in the realized structure sufficiently changes incentives to permanently disrupt an existing social structure, 


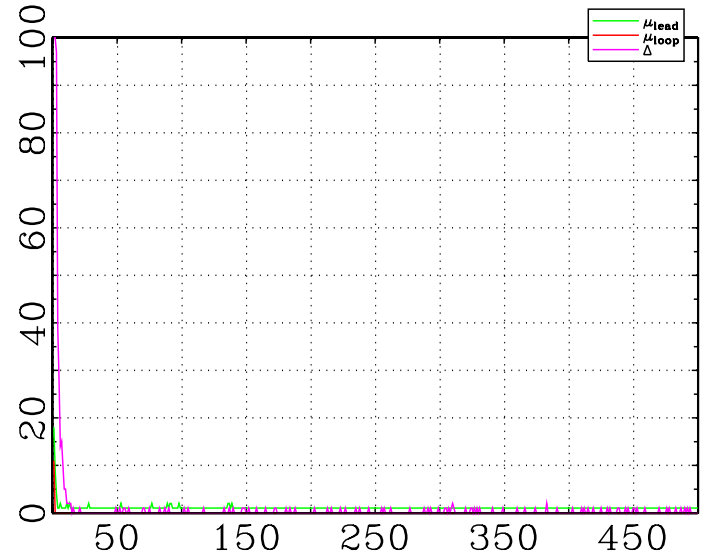

(a) Time-series of $\left\{\mu_{\text {lead }}, \mu_{\text {loop }}, \Delta\right\}=\{$ number lead, number looped, inefficiency $\}$.

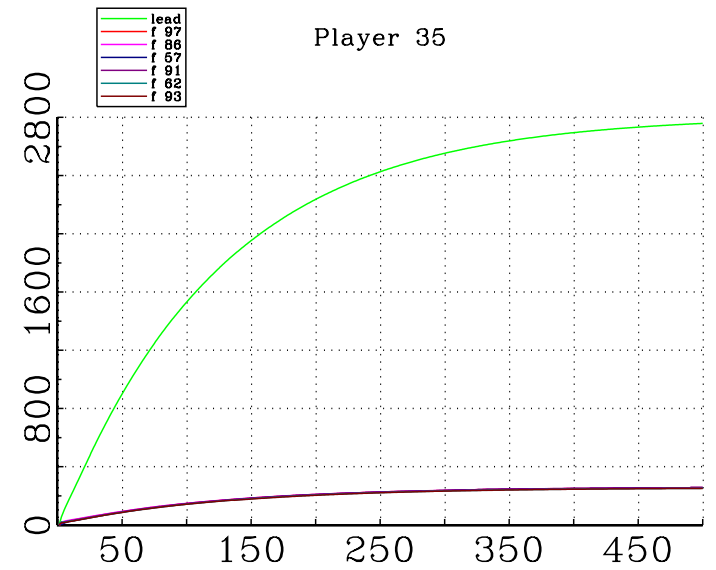

(c) Time-series $A_{0}-A_{6}$ for the eventual leader.

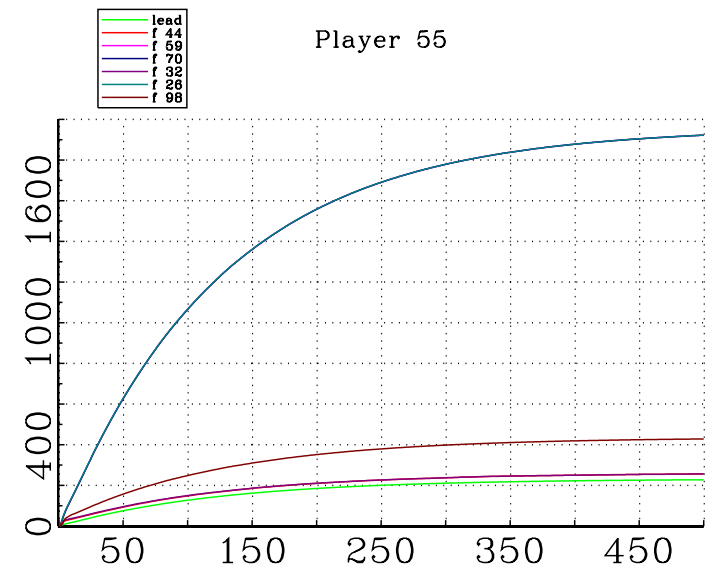

(e) Time-series $A_{0}-A_{6}$ for an agent two links from the leader.

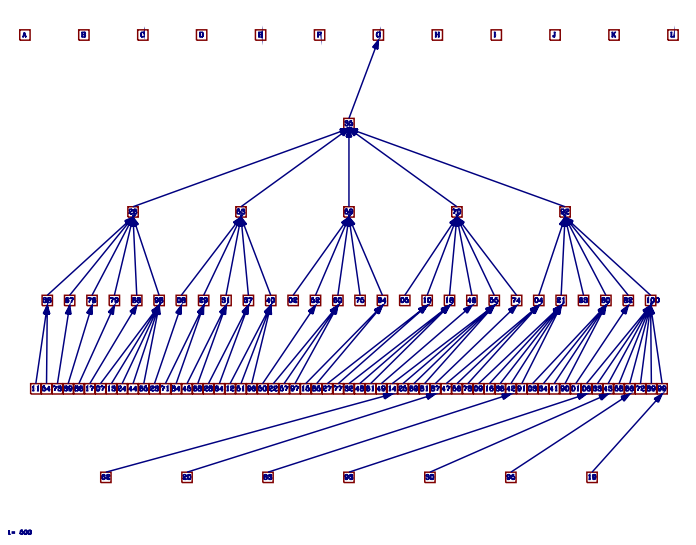

(b) Social structure in the final period $(T=500)$.

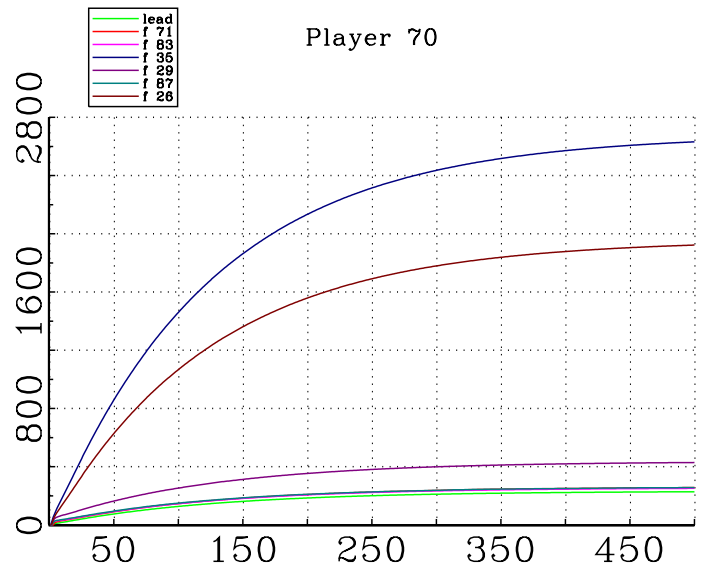

(d) Time-series $A_{0}-A_{6}$ for an agent one link from the leader.

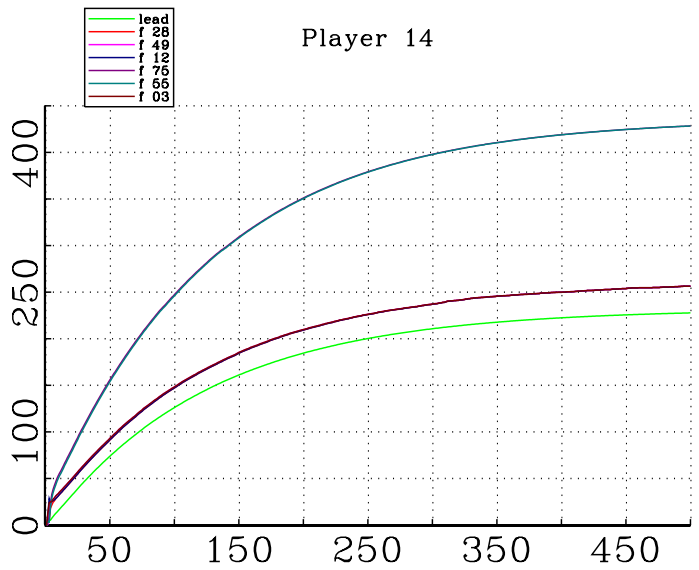

(f) Time-series $A_{0}-A_{6}$ for an agent most distant from the leader.

Figure 6: Lock-in: $\kappa_{l}=\kappa_{f}=0.2$. The compounding of evidence allows the most distant followers to recognize the superiority of following. 
allowing a new leader to emerge.

The leader at the end of the example presented in Figure 7 only came to be leader around $t=380$. Prior to this, there had been other leaders, including the agent depicted in Figure $7 f$.

\subsubsection{Reinforcement}

A tendency towards the use of reinforcement learning potentially undermines social adaption necessary to generate the equilibrium structure. The evolution works when the population adjusts towards historically high paying alternatives. With reinforcement learning the agents remain loyal to any action with a positive payoff, generally missing opportunities for improvement, particularly as they might arise in a changing social structure.

Treatment 6 introduces reinforcement learning in the decision to lead. Discounting the counterfactual performance associated with leading by an agent who follows, as is the case with $\delta_{l}<1$, improves the ability to generate the equilibrium structure. Under-utilization of the lead option among followers, due to the discount, is beneficial for the evolution towards, and continued stability of, the social structure. As was the case with the baseline treatment, the one failure to produce the equilibrium structure with $\delta_{l}=0.2$ is the consequence of a group of followers producing a self-referencing loop for the period. Even a small discount goes a long way to increasing instances of full cooperation with, for example, $\delta_{l}=0.8$ producing the equilibrium structure in $88 \%$ of the simulation runs.

Treatment 7 introduces reinforcement learning in the decision to follow. Discounting the counterfactual performance associated with following is twice detrimental for building the equilibrium structure. Too many agents continue to lead when they discount following as an option. Further, empowered by their followers, multiple hierarchies can persist if followers discount the benefit of switching to a larger hierarchy. The population presented in Figure 8 experiences early success in identifying and following a single leader. The followers slowly dissipate. With a small differential between being the most distant follower and leading, once an agent leads, the discount applied to the evidence of the reward to following tends to discourage ever returning.

The example in Figure 8 is one of the roughly $50 \%$ of the runs ending with less than 50 followers. The other runs appear to stabilize at the locally stable disequilibrium with two tiers of reliable followers, as exemplified in Figure 9. 


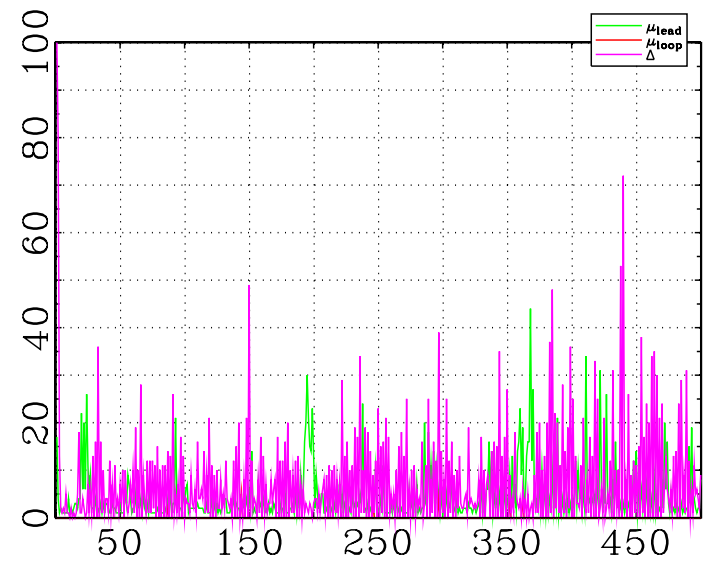

(a) Time-series of $\left\{\mu_{\text {lead }}, \mu_{\text {loop }}, \Delta\right\}=\{$ number lead, number looped, inefficiency $\}$.

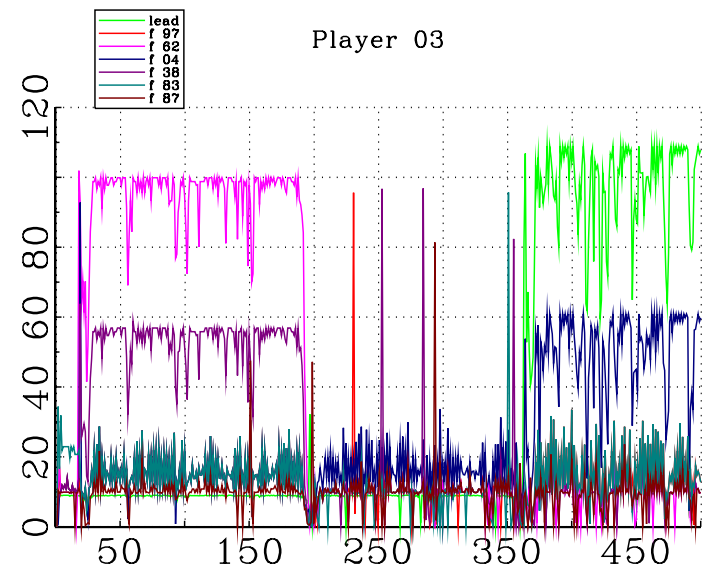

(c) Time-series $A_{0}-A_{6}$ for leader in terminal structure. Only began leading after $t=350$.

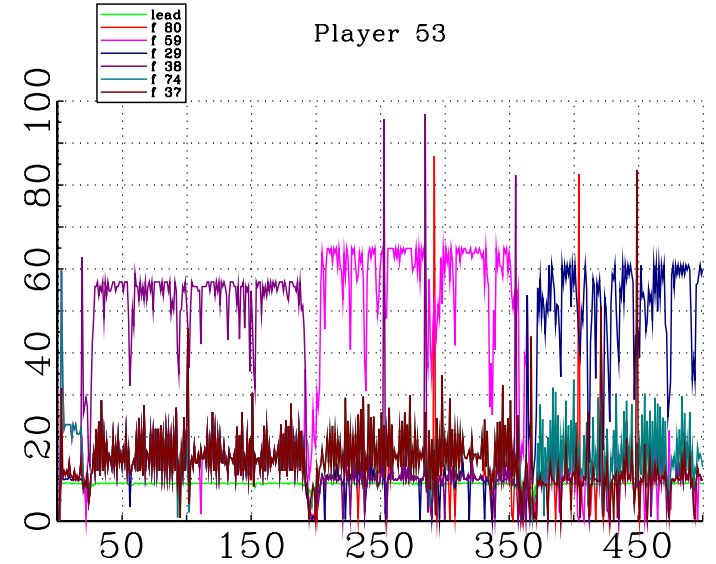

(e) Time-series $A_{0}-A_{6}$ of agent two links from leader in terminal structure.

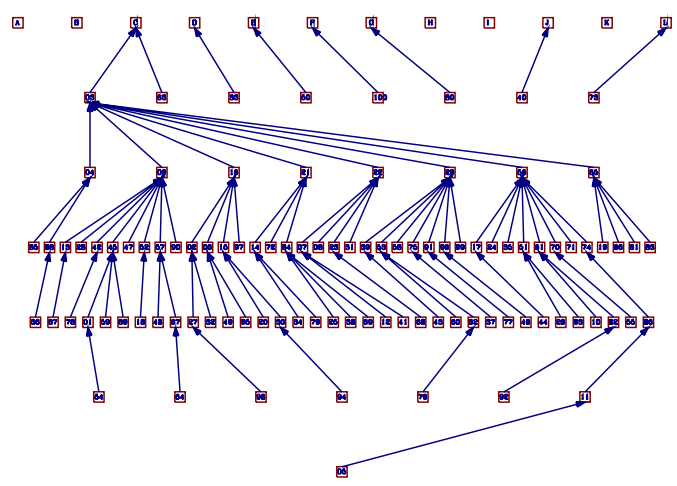

(b) Social structure in the final period $(T=500)$. Full participation but high inefficiency.

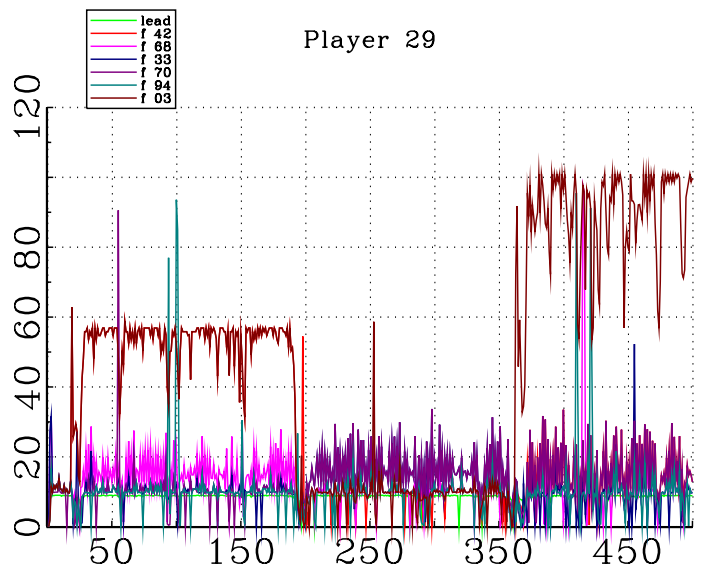

(d) Time-series $A_{0}-A_{6}$ for an agent one link from the leader in terminal tructure

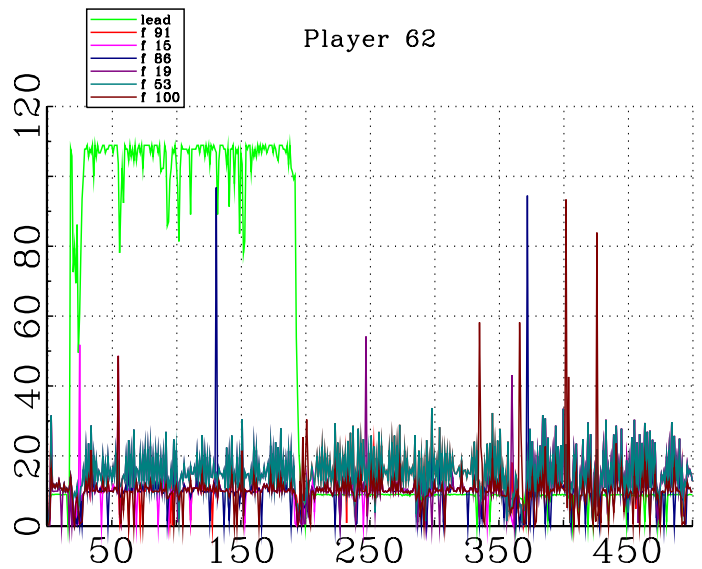

(f) Time-series $A_{0}-A_{6}$ of agent three links from leader in terminal structure. Was leader $t \in[20,200]$.

Figure 7: High Recency: $\phi_{f}=\phi_{l}=0$. With no personal memory, all social memory is embedded in the network. The indecisiveness among the most distant followers can lead to disruptions in the social structure sufficient to bring about a new leader. 


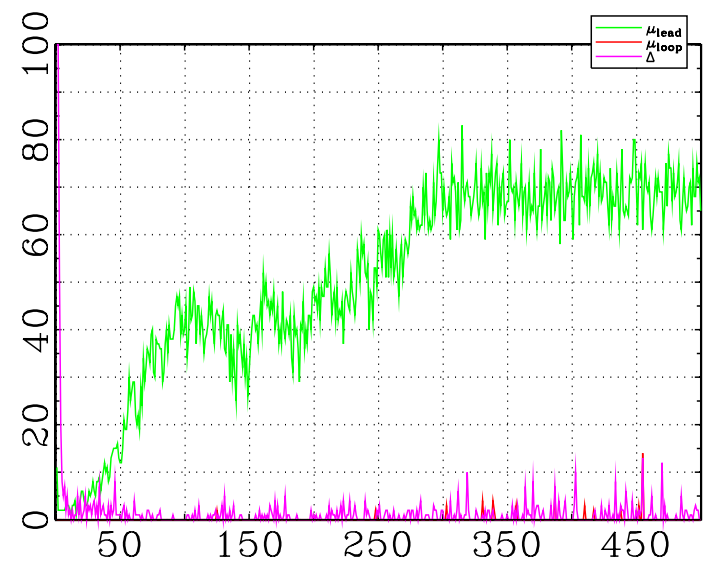

(a) Time-series of $\left\{\mu_{\text {lead }}, \mu_{\text {loop }}, \Delta\right\}=\{$ number lead, number looped, inefficiency $\}$. Early near equilibrium structure dissipates.

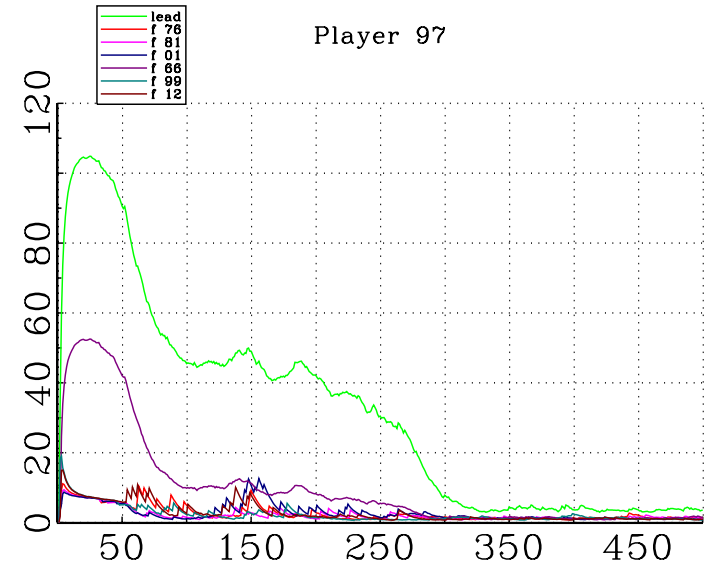

(c) Time-series $A_{0}-A_{6}$ for the early leader.

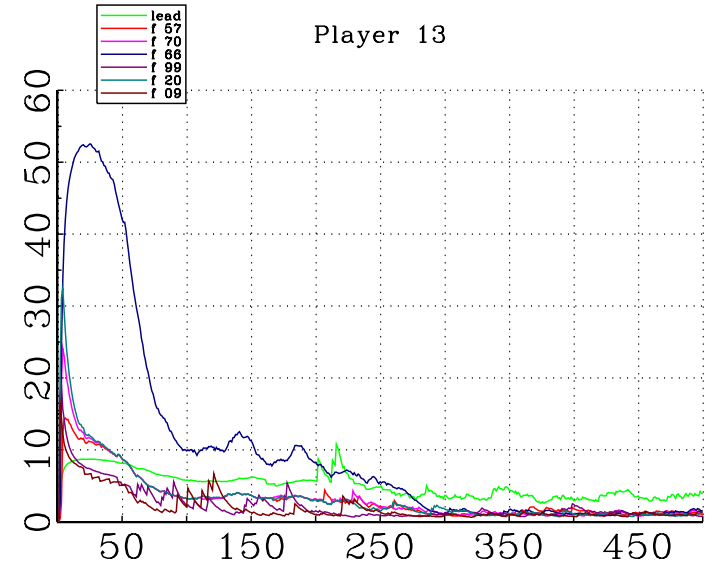

(e) Time-series $A_{0}-A_{6}$ for an agent two links from the leader.

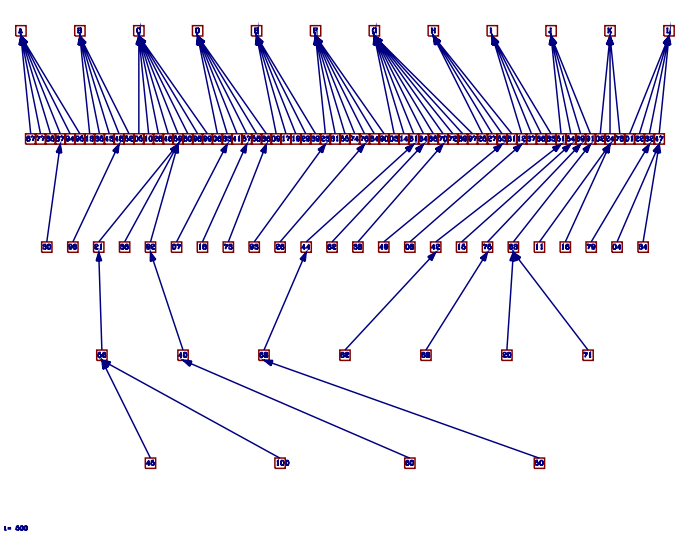

(b) Social structure in the final period $(T=500)$ produced from an absence of regularity in following.

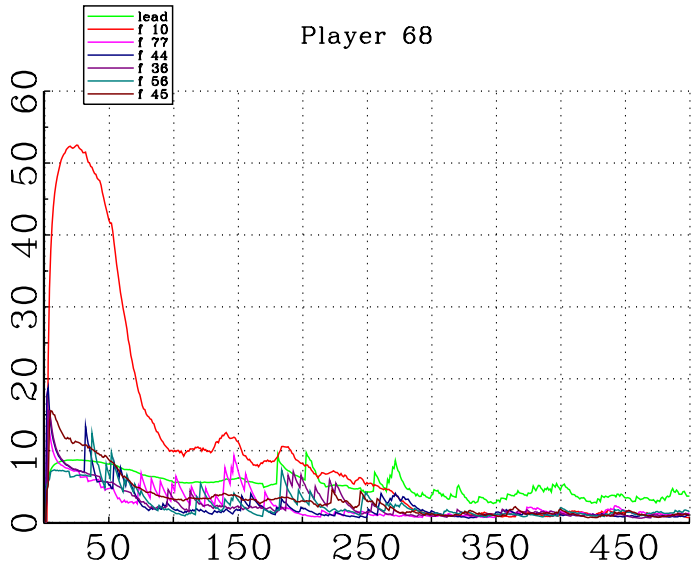

(d) Time-series $A_{0}-A_{6}$ for an agent one link from the leader.

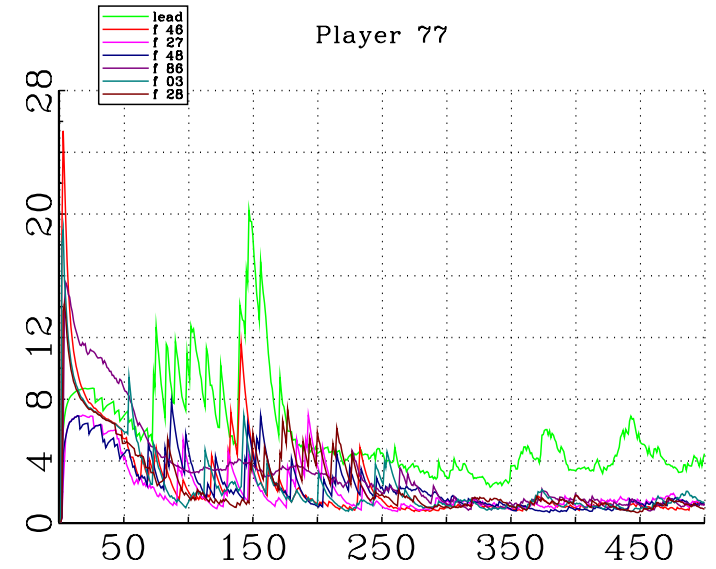

(f) Time-series $A_{0}-A_{6}$ for an agent most distant from the leader.

Figure 8: Reinforcement learning in follow decision: $\delta_{f}=0.7$. Agents fail to fully recognize the benefits to following when they lead. When those most distant from the leader experiment with leading, the discounting reduces the likelihood of a return. 


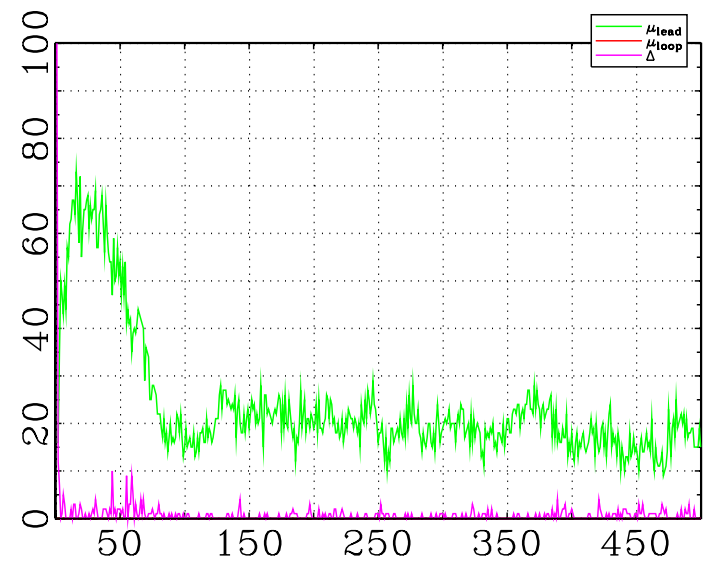

(a) Time-series of $\left\{\mu_{\text {lead }}, \mu_{\text {loop }}, \Delta\right\}=\{$ number lead, number looped, inefficiency $\}$ Local stability achieved with two tiers of followers.

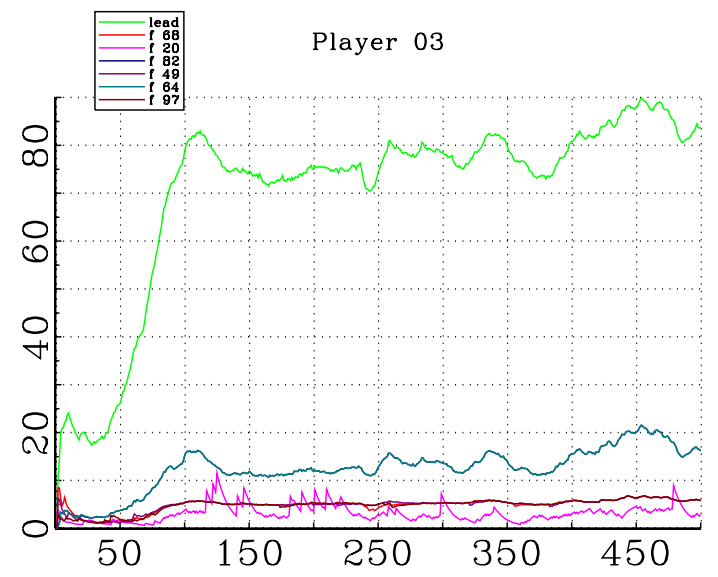

(c) Time-series $A_{0}-A_{6}$ for the eventual leader.

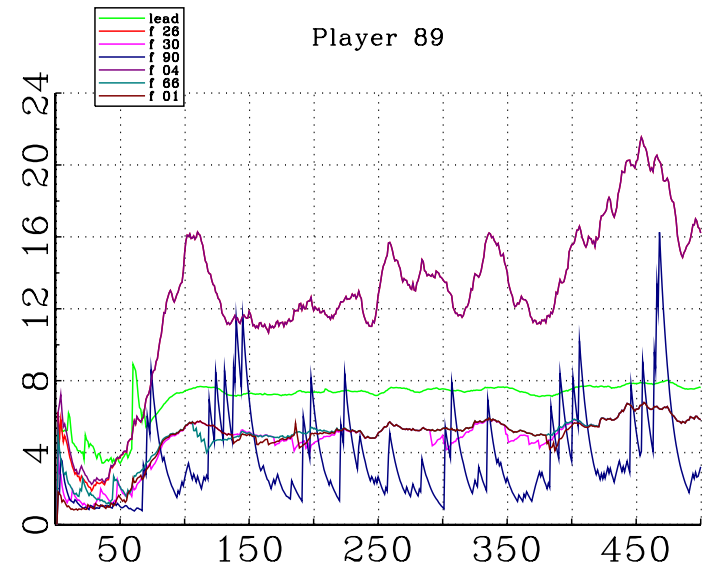

(e) Time-series $A_{0}-A_{6}$ for an agent two links from the leader.

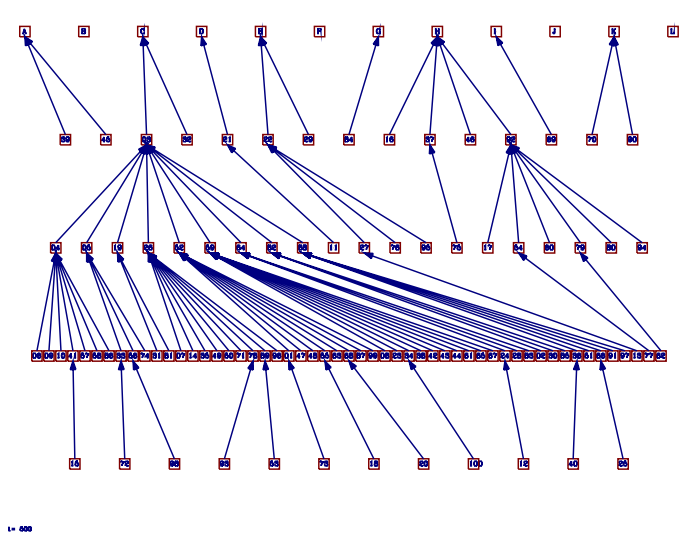

(b) Social structure in the final period $(T=500)$. Two stable tiers of followers. Indecisiveness in 3rd tier.

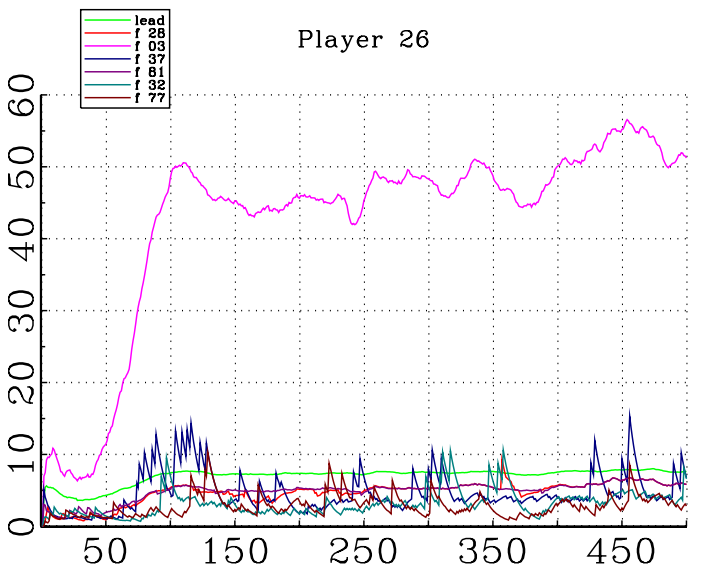

(d) Time-series $A_{0}-A_{6}$ for an agent one link from the leader.

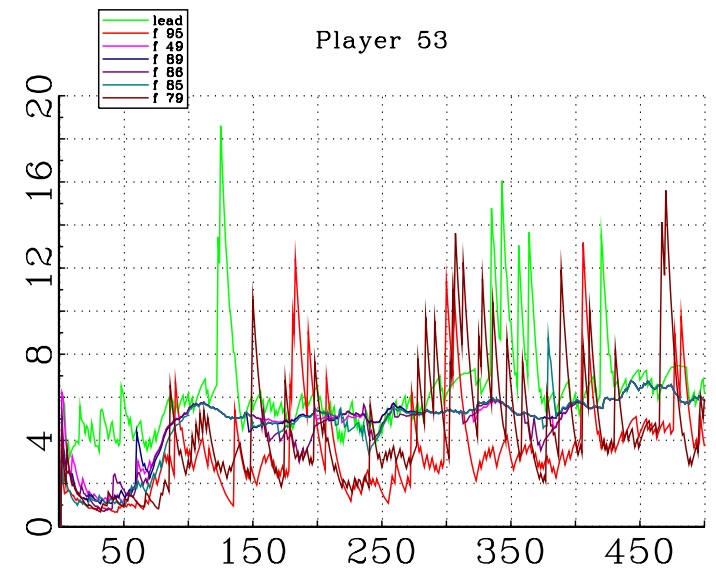

(f) Time-series $A_{0}-A_{6}$ for an agent who leads. Reinforcement biases perceived performance in favor of leading.

Figure 9: Reinforcement learning in follow decision (alternate): $\delta_{f}=0.7$. Population settles at locally stable disequilibrium structure. Leader commands two tiers of reliable followers. 


\subsubsection{Independence and bias}

Recall from Figure 2 that $\lambda$ and $A_{0,0}$ profoundly effect the structure realized in the first period. For $\lambda=1$, the agent treats each option equally and as though it were independent from each of the other options. Suppose $A_{0}=\cdots=A_{d}$ and $\lambda=1$, then $\rho_{i}=(1-\rho) w=1 /(d+1)$. If one option exhibits higher performance, it draws probability weight away from the other options equally. For $\lambda \rightarrow 0$, a parent decision is between lead or follow. Identifying who to follow is a descendent of the follow decision. With $A_{0}=\cdots=A_{d}$ the two options in the two parent decision options are given equal weight so that $\rho_{i}=1 / 2$. Each option in the dependent decision about who to imitate gets equal weight within the follow option so that $w_{i}^{j}=1 / d$. If one contact outperforms the others, it primarily absorbs probability weight from the other contacts. It also absorbs probability weight from the lead option to the extent that the aggregate performance associated with following increases with the additional probability weight on the better performing contact.

Setting $A_{0,0}>0$ introduces a predisposition to lead. In this case, agents only switch to follow as experience gathers indicating that the lead option does not warrant the initial bias. ${ }^{5}$

Treatment 8 introduces nesting with $\lambda=0.15$. The run is typical in achieving stability at the disequilibrium consisting of two tiers of regular followers, as seen in Figure 10b. The example in Figure 10 is somewhat atypical in that there is early success in approaching the equilibrium structure. Followers proceed to abandon the hierarchy starting around period 400 . When the population begins to return to following, it is under a new leader.

With nesting, leading receives a greater share of the probability weight when there is little performance differential between leading and following. This leads to a greater proportion of the most distant followers experimenting with leading (seen in the fist 400 periods of Figure 10f in the greater frequency of dips and spikes), creating greater disruption to any regular structure.

With $A_{0,0}=3.5$, employed in Treatment 9 , roughly $80 \%$ of the population leads in the first period. For the typical agent, $A_{0, t}(i)$ would be expected to decay over time as the initial value of the lead performance measure exceeds the expected payoff when $\rho=0.8$.

The initial bias produces greater homogeneity in the early periods, slowing the identification of a leader. The reported frequencies of Table 1 and individual progressions suggest that the initial condition may impact who emerges as leader but does not much effect the process. This is investigated further in Section 3.3

\footnotetext{
${ }^{5}$ In experiments on the same setting, 80 percent of subjects lead in the first period.
} 


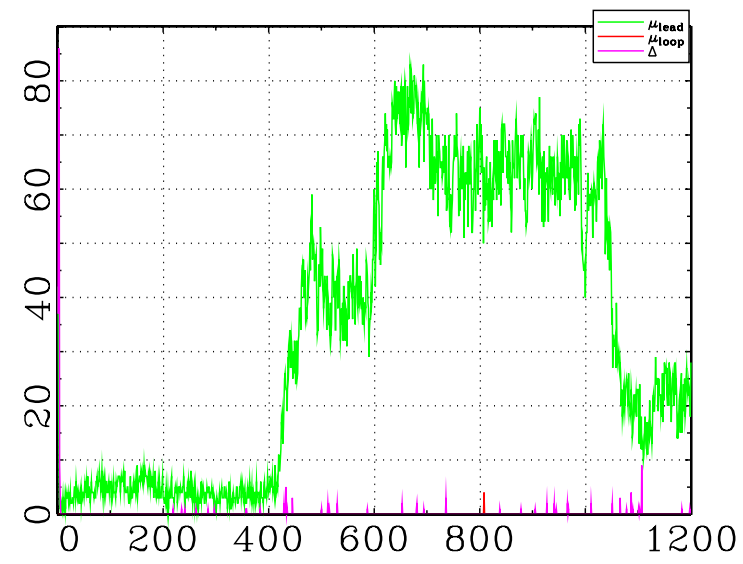

(a) Time-series of $\left\{\mu_{\text {lead }}, \mu_{\text {loop }}, \Delta\right\}=\{$ number lead, number looped, inefficiency $\}$

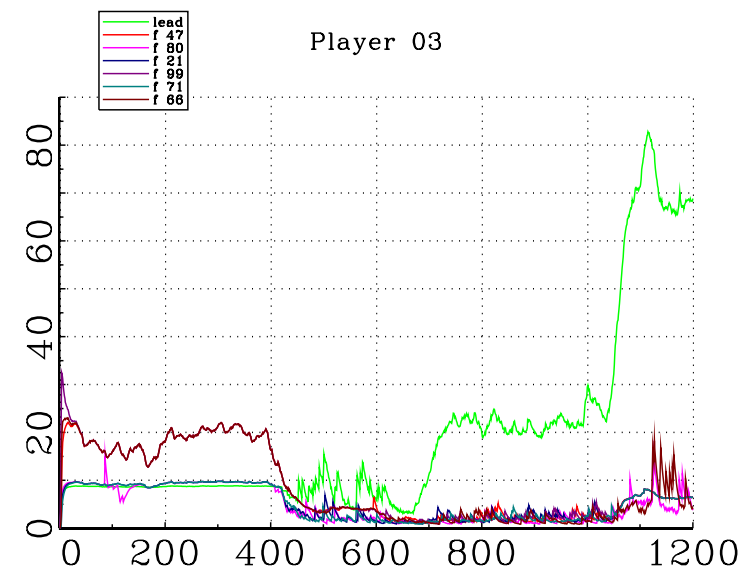

(c) Time-series $A_{0}-A_{6}$ for the eventual leader.

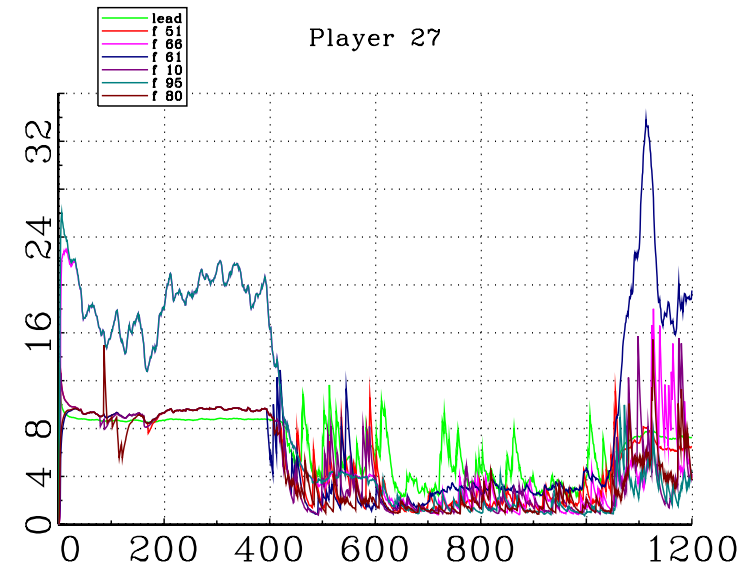

(e) Time-series $A_{0}-A_{6}$ for an agent two links from the leader.

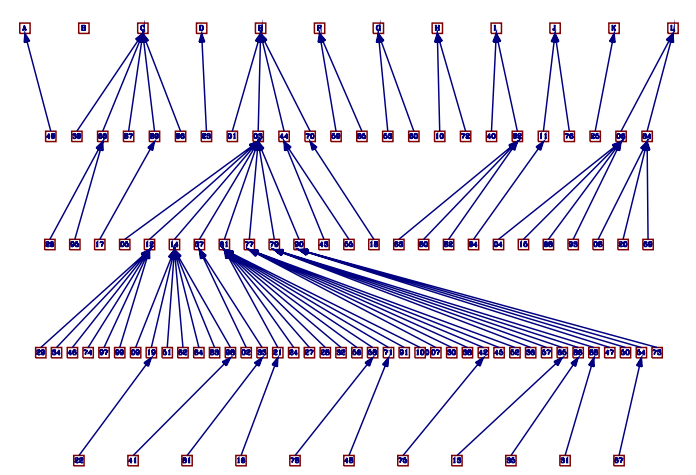

$\cdots$

(b) Social structure in the final period $(T=1200)$. Two tiers of reliable followers

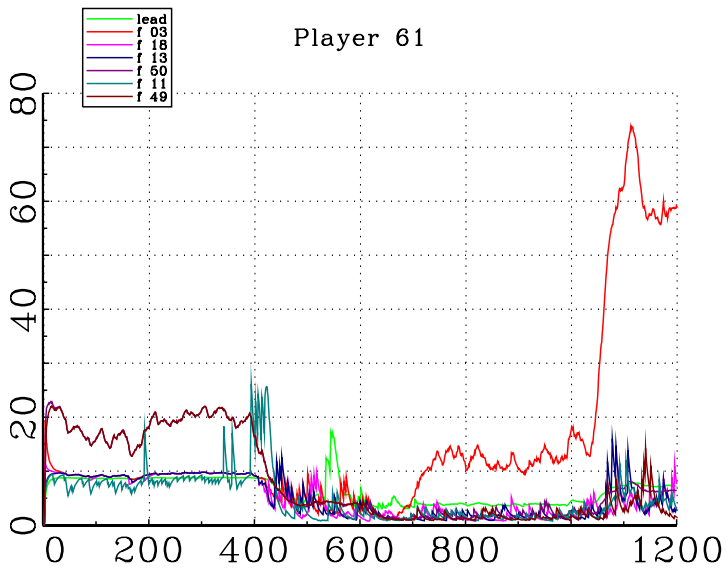

(d) Time-series $A_{0}-A_{6}$ for an agent one link from the leader.

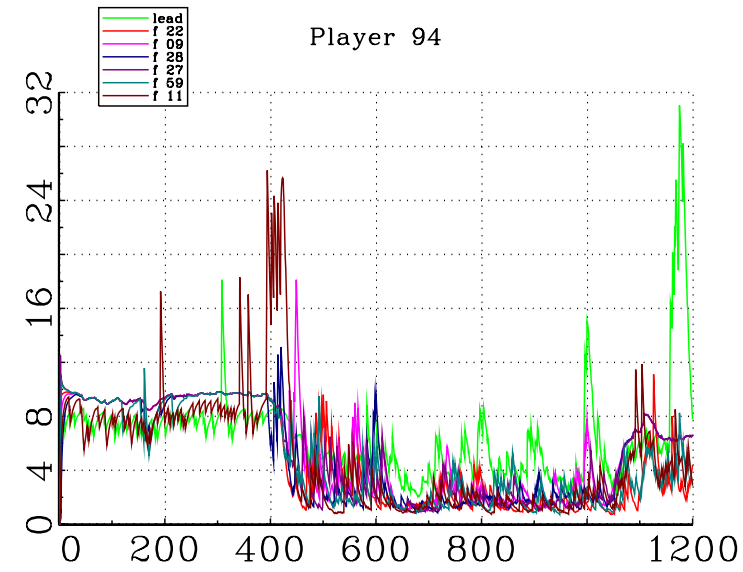

(f) Time-series $A_{0}-A_{6}$ for an agent most distant from the leader.

Figure 10: Nesting: $\lambda=0.1$. The population cycles through each of the locally stable disequilibria. The terminal leader was not the original leader through the first 400 periods. The figures show out to $t=1200$ but the final structure remains in place through $T=5000$. 


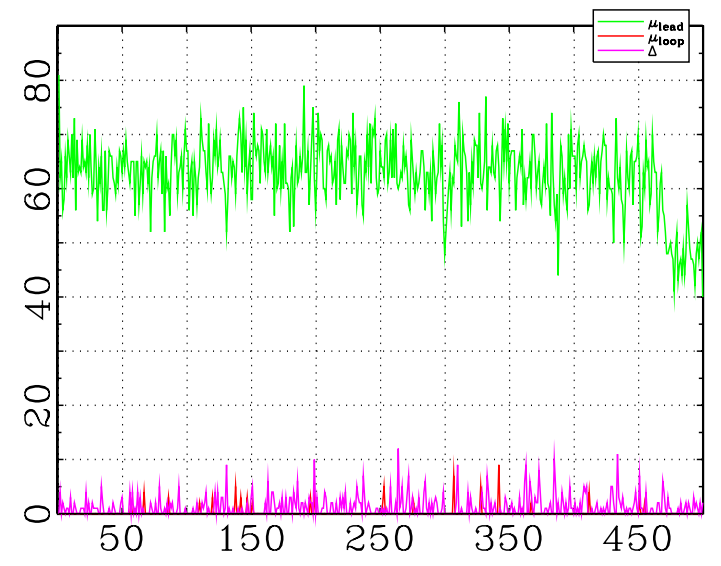

(a) Time-series of $\left\{\mu_{\text {lead }}, \mu_{\text {loop }}, \Delta\right\}=\{$ number lead, number looped, inefficiency $\}$

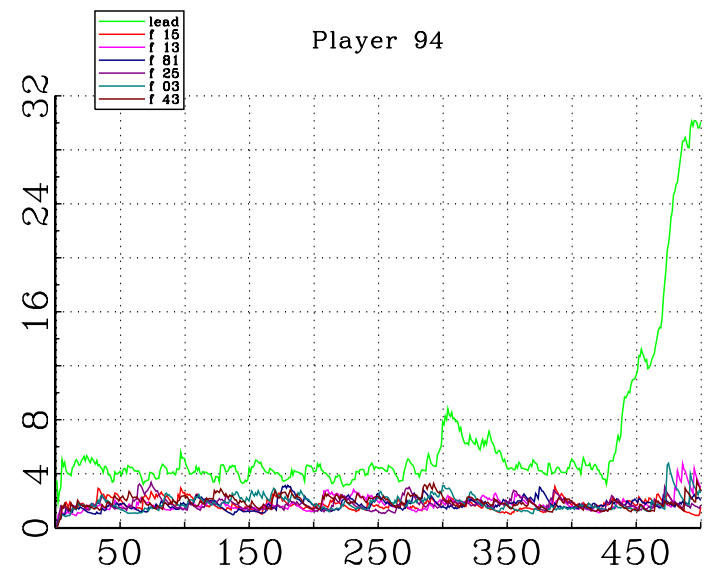

(c) Time-series $A_{0}-A_{6}$ for the eventual leader.

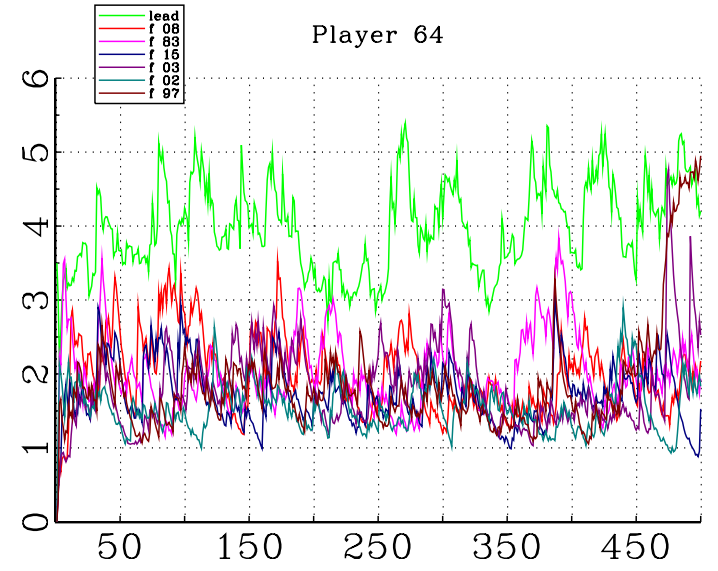

(e) Time-series $A_{0}-A_{6}$ for an agent two links from the leader.

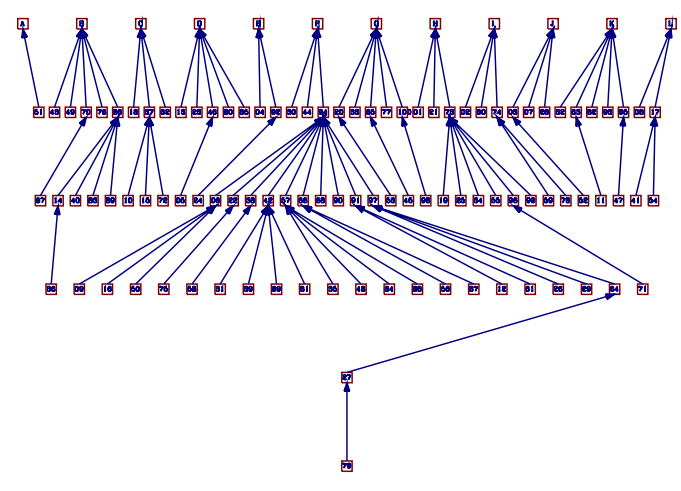

(b) Social structure in the final period $(T=500)$. One tier of reliable followers

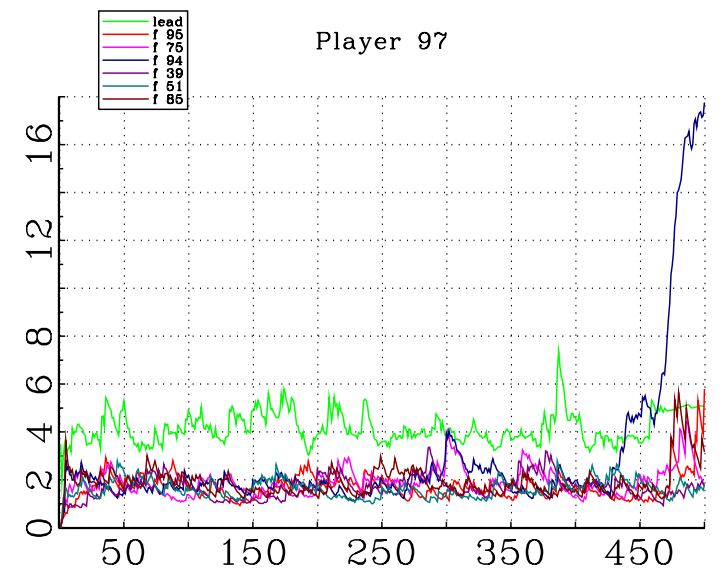

(d) Time-series $A_{0}-A_{6}$ for an agent one link from the leader.

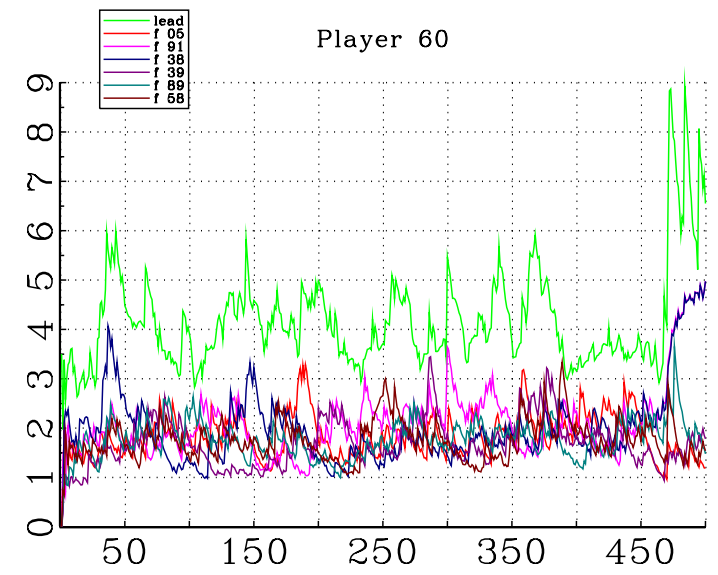

(f) Time-series $A_{0}-A_{6}$ for an agent otherwise 3 links from leader.

Figure 11: Predisposition to lead: $A_{0,0}=3.5$ The initial bias produces $\mathrm{h}$ 


\subsubsection{Locally stable disequilibrium}

In simulation, populations spend considerable durations within a small number of apparently stable disequilibrium states. The time-series in 10a shows a population cycling through a number of locally stable states of disequilibrium. Figure 12 shows example structures sampled from each state. The explanation for the locally stable states of disequilibrium comes from considering stable social organizations in which some or all of the population employ a mixed strategy.

Consider a homogeneous population employing a mixed strategy in which the probability of leading is $\rho_{i}=\rho$ and $w_{i, j}=(1-\rho) / n_{d} \forall i, j \in N_{i}^{d}$. This describes the behavior generating the structure observed in Figure 12a. In each period a randomly determined portion $\rho$ of the population leads while $(1-\rho)$ follows, generating no real social organization. This behavior generates expected payoffs, ${ }^{6}$

$$
\begin{aligned}
\pi_{l}^{e}(\rho)=\mathbb{E}\left(\pi_{l}(\rho)\right) & =\left(a_{J}+(1-\rho) a_{T}\right) \frac{n}{m} \\
\pi_{f}^{e}(\rho)=\mathbb{E}\left(\pi_{f}\right) & =a_{J} \frac{n}{m} .
\end{aligned}
$$

Recall that, for $\phi_{h}<1$ or $\phi_{h}=1$ and $\kappa_{h}=1, h=l, f$, the performance measure reaches a steady state as indicated in (8). Let $\alpha_{h}=\left(1-\phi_{h}\left(1-\kappa_{h}\right)\right) /\left(1-\phi_{h}\right)$. The mixed strategy fixed point is the $\rho_{0}^{*}$ that solves, based on the nested logit condition in (4),

$$
\rho=\frac{e^{\mu \alpha_{l} \pi_{l}^{e}(\rho)}}{e^{\mu \alpha_{l} \pi_{l}^{e}(\rho)}+n_{d}^{\lambda} e^{\mu \alpha_{f} \pi_{f}^{e}(\rho)}}
$$

Since $\pi_{l}^{e} \neq \pi_{f}^{e}$, the mixed-strategy fixed point is not an equilibrium. The superior performance to leading ensures that leading receives more than an equal share of the probability mass.

The other stable disequilibrium structures depicted in Figure 12 involve heterogeneous behavior made up of four position types. These are

1. $\{i\}$ : a leader, with $\rho_{i}=1$,

2. $N^{D}(i)=\left\{j \in N \mid \underline{d}_{j, i}=D\right\}$ : a population of mixing agents with $\rho_{D} \in(0,1)$ who, when they follow, are at distance $D$ from the leader,

3. $N^{R}(i, D)=\left\{j \in N \backslash\{i\} \mid \underline{d}_{j, i}<D\right\}$ : a population of reliable followers, with $\rho_{R}=0$, for all $j \in N \backslash\{i\}$ capable of $d_{j, i}<D$, and

\footnotetext{
${ }^{6}$ For computation convenience, assume that all followers link with someone who leads in the period. This is not necessarily true as the chosen contact may have also chosen to follow. As a consequence, the actual expected payoff to following is higher than indicated by this formula.
} 


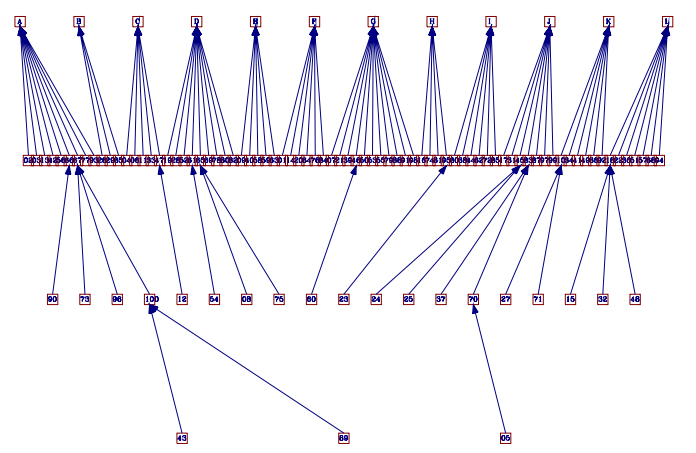

(a) $t=650$, homogeneous population of mixing agents.

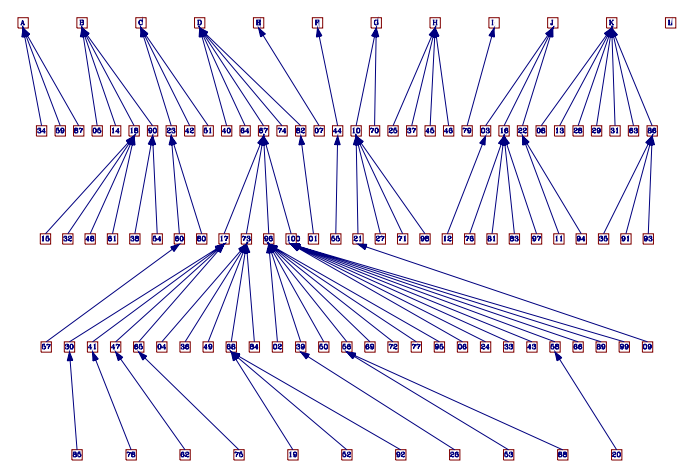

(c) $t=510$, mixing at distance $D=3$. Leader has few direct followers.

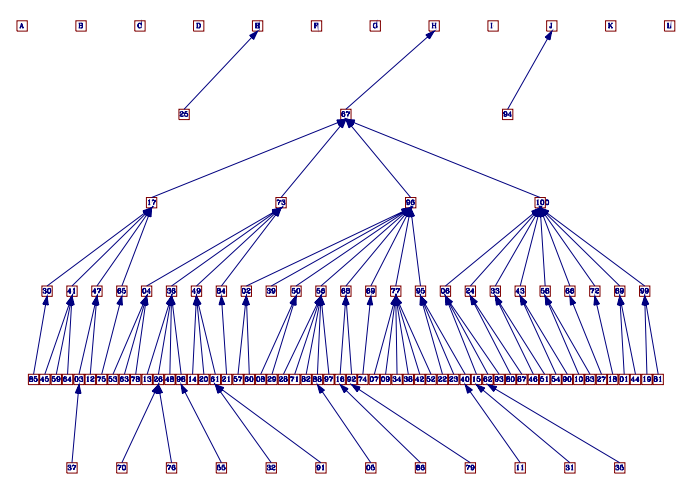

(e) $t=200$, mixing at distance 4 (bottom tier).

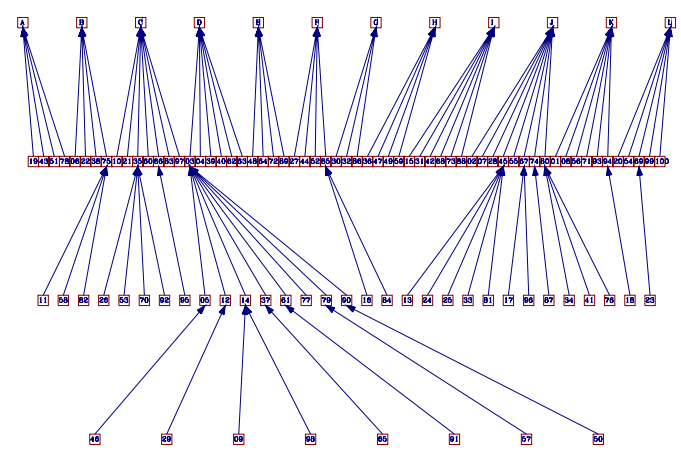

(b) $t=810$, mixing at distance $D=2$.

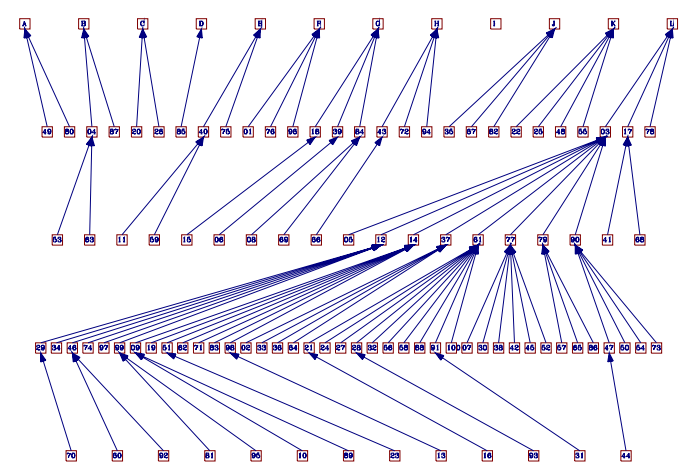

(d) $t=1200$, mixing at distance $D=3$. leader with many direct followers.

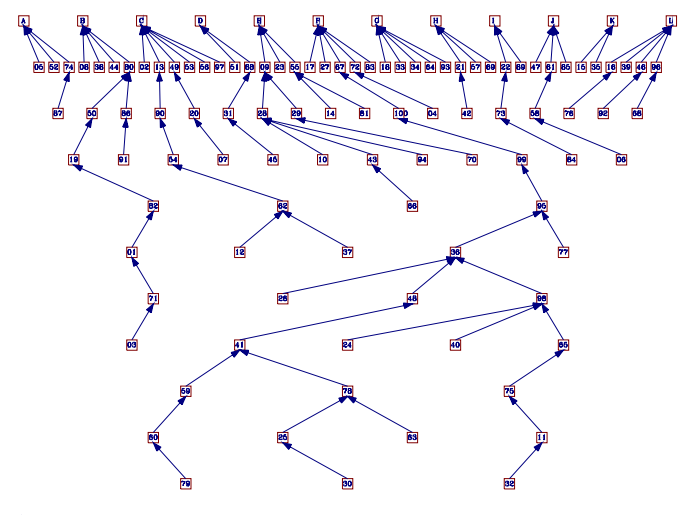

(f) $t=1$ Random action

Figure 12: Equilibrium and disequilibrium structures from the simulation producing Figure 10. 
4. $N^{l}(i, D)=\left\{j \in N \underline{d}_{j, i}>D\right\}$ : a population that leads, with $\rho_{l}=1$, for all $l \in N$ incapable of $d_{j, i} \leq D$.

Let $n_{D}, n_{R}$, and $n_{l}$ represent, respectively, the size of the $N^{D}(i), N^{R}(i, D)$, and $N^{l}(i, D)$ populations.

For those employing the mixed strategy,

$$
\begin{aligned}
\mathbb{E}\left(\pi_{f}(\rho, D)\right) & =a_{J}\left(1+n_{R}+(1-\rho)\left(n_{D}-1\right)+\frac{1}{m}\left(\rho\left(n_{D}-1\right)+n_{l}\right)\right) \\
\mathbb{E}\left(\pi_{l}(\rho, D)\right) & =\frac{1}{m}\left(a_{J}(n-1)+a_{T}\left(n_{R}+(1-\rho)\left(n_{D}-1\right)\right) .\right.
\end{aligned}
$$

Let

$$
C=\theta-\frac{n_{R}+(1-\rho)\left(n_{D}-1\right)}{1+n_{R}+(1-\rho)\left(n_{D}-1\right)}
$$

The condition $C \geq 0$ implies $\mathbb{E}\left(\pi_{f}(\rho, D)-\pi_{l}(\rho, D)\right) \geq 0$. Since $n_{R}+(1-\rho)\left(n_{D}-1\right)<n-1, B>0$ implies $C>0$ for all $\rho$. Thus, there is no value of $\rho$ that will produce $\mathbb{E}\left(\pi_{f}(\rho, D)-\pi_{l}(\rho, D)\right)=0$. In addition, the fixed point assigns greater probability mass on following relative to the probabilities produced by indifference.

Let $\rho_{D}^{*}$ indicate the value of (11) evaluated at expected payoffs (12) and (13). The condition indicates that the profit differential between the $\rho_{D}^{*}$-dependent lead and follow payoffs for the $N^{D}(i)$ population support the $\rho_{D}^{*}$ probability to lead.

To sustain the structure, the mixing population must support the pure strategy behavior of the other agents. In particular, the $\left(1-\rho_{D}^{*}\right) n_{D}$ averaged sized population following at distance $D$ has to provide enough of a preemption payoff to the $D-1$ distance followers such that $\rho_{R}=0$. Transitions to a smaller hierarchy are likely the result of small realized populations at distance $D$ offering insufficient reward, leading the $D-1$ distance followers to mix. Similarly, as $\rho_{D}^{*} \rightarrow 0$, the agents in the $N^{l}$ population have reasonably reliable connections to the leader, making following sufficiently attractive to start mixing. Transitions to a larger hierarchy are likely the result of persistent following on the part of the $N^{D}(i)$ population such that they attract their own followers. Following becomes more attractive to everyone in the $N^{D}(i)$ population as the $D+1$ tier fills in.

Table 2 identifies numerically derived $\rho_{D}^{*}$ values based on the parameters of Treatment 1 . Using a symmetric network of $n_{d}$ each of inlinks and outlinks for each agent, then $n_{D}=\min \left\{n_{d}^{D}, n-1-n_{R}\right\}$. For $n_{d}=6$ and $n=100$, the maximum distance possible is three links. The large $N^{l}(i, 2)$ population unable to link to the leader contributes to the large number of agents who lead in the $D=2$ mixing state. 
Table 2: Mixing behavior and resulting social structure. Numerical solutions based on a symmetric contact network using parameters from Treatment 1 . Also based on each agent at distance $D$ having only one contact at the $D-1$ distance.

\begin{tabular}{|c|c|c|c|}
\hline Mixing distance & $n_{R}$ & $\mathbb{E}\left(\mu_{\text {lead }}\right)$ & $\rho_{D}^{*}$ \\
\hline \hline $0 / 1$ & 0 & 67 & 0.67 \\
\hline 2 & 6 & 72 & 0.40 \\
\hline 3 & 36 & 12 & 0.32 \\
\hline
\end{tabular}

\subsubsection{Strong desire to lead $(B<0)$}

A decrease in $\theta=(m-1) \frac{a_{J}}{a_{T}}$ such that $B<0$ changes the incentives to the most distant followers. Decreasing $m$ improves the likelihood that leading will produce a match with the dominant leader, increasing the expected payoff to the strategy. A decrease in $\frac{a_{J}}{a_{T}}$ increases the relative premium reward to leading. Both changes increase the incentive to lead, and for $B<0$ the incentive is enough to induce the most distant followers of the dominant leader into leading.

The $B<0$ equilibrium structure, with a hierarchy of size $n_{s}<1-n$ followers, is potentially more difficult to achieve. With an interior $n_{s}^{*}$ there is always some agents on the margin between leading and following. An additional frailty arrises from the property that, to remain an attractor, for $B<0$ the equilibrium structure requires the presence of only one leader. In the presence of multiple leaders, autarky is the attractor with the most distant followers in each hierarchy preferring to lead. ${ }^{7}$

Simulations of Treatment 10, with $B=-0.055$, produced $n_{s} \geq 50$ in $100 \%$ of the treatments despite an $n_{s}^{*}=15$. An examination of the simulation output suggests roughly equal performance between the lead and follow options. The simulations identify the existence of an mixed strategy equilibrium that is Pareto improving to the pure strategy equilibrium supporting $n_{s}=n_{s}^{*}$.

Let $\bar{d}_{i}$ represent the maximum distance from leader $i$ for $\sigma \in \Sigma^{*}, \bar{d}_{i}=\max _{j \in N^{S}(i)} d_{i, j}$. Follower $j$ with $d_{j, i, t}=\bar{d}_{i}$ can increase her distance (employing an inefficient link), increasing the payoff to any other follower at distance $\bar{d}_{i, t}$ without lowering her own payoff. Whether at $\bar{d}_{i}$ or $\bar{d}_{i}+1$, she is the most distant follower, receiving only the conformity reward. Relative to the efficient structure, there is no loss. When all agents at $\bar{d}_{i}$ employ a non-zero probability of locating themselves at distance $\bar{d}_{i}+1$, the cost of realizing the greater distance is the lost opportunity to benefit from the other players who have also increased their distance.

There is a locally stable fixed point at which each of the distance- $\bar{d}_{i}$ followers employs a non-zero probability of increasing her distance. The same is true for each tier when it is the most distant of the followers. The practice of mixing by the most distant followers increases the payoff to the entire cohort sufficiently to

\footnotetext{
${ }^{7}$ see Goldbaum (2016).
} 
exceed the payoff to leading. Their membership in the hierarchy supports the membership of all the agents above them. The mixing behavior is evident in the inefficiency recorded in Figure 13a.

For $B>0$, the pure strategy equilibrium is superior to the mixed strategy disequilibrium. Everyone, including the most distant follower, is better off receiving the certain conformity reward inherent in the equilibrium structure. For $B<0$, the mixing is Pareto improving over the pure strategy equilibrium as the mixing support a larger hierarchy generating greater conformity and more opportunities to preempt others.

A tendency to lock-in, introduced in Treatment 11, can disrupt the mixed-strategy equilibrium. It magnifies performance differentiation, creating greater consistency in the relative positioning and decreases the number who employ an inefficient link within the hierarchy. The frequencies reported for Treatment 11 reveal that a still large number of runs remain at the mixed strategy equilibrium. Those that disperse typically end either near the equilibrium structure, as is the case in Figure 14, or reach the homogeneous mixing disequilibrium.

\subsection{Leadership Characteristics}

\subsubsection{Social advantage}

Visibility should impact on the evolutionary process. A randomly generated contact network produces a non-degenerative distribution of incoming links for each agent. Let $e_{i}$ be the number of contact links, captured in $g$, directed at agent $i$. As revealed in Figure 15, those with a greater number of incoming links experience a heightened probability for emerging as a leader. The two figures are each the product of 5,000 simulation iterations. In each figure, the solid (blue) curve is the distribution over the entire population of 500,000 agents as initiated at the start of the treatment. The long-dashed (red) curve is the distribution of inlinks among the ex post population of dominant leaders as realized at the end of each iteration. The short-dashed (black) curve is the conditional probability of emerging as a leader based on the agent's $e_{i}$. The two treatments employ different behavior parameters but the same randomly generated 5,000 networks of contacts upon which to consider behavior.

The treatment presented in Figure 15a employs the baseline parameters. Recalling Figure 2a, the considerable heterogeneity in realized payoffs in the first period facilitates quick identification and emergence of the equilibrium structure, typically in fewer than five periods. The random realization of potentially long following chains generates vastly different payoffs in the first round that are quickly incorporated into an imitation network. Advantage comes from the greater visibility and the greater chance of having followers in that first period. 


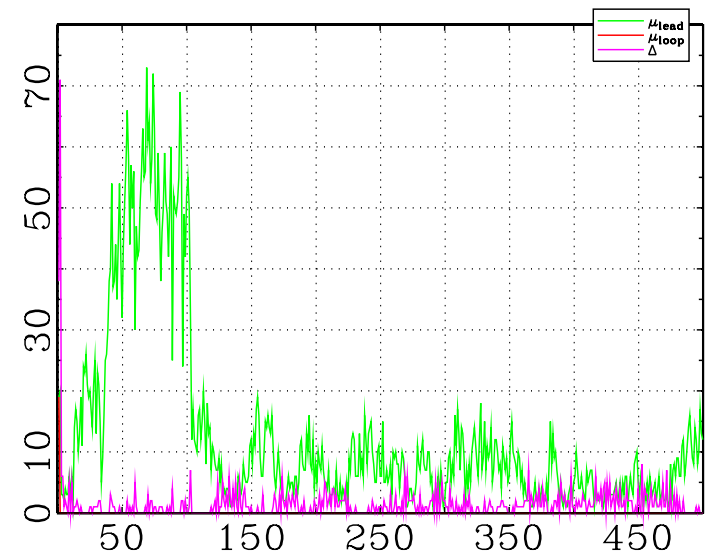

(a) Time-series of $\left\{\mu_{\text {lead }}, \mu_{\text {loop }}, \Delta\right\}=\{$ number lead, number looped, inefficiency $\}$

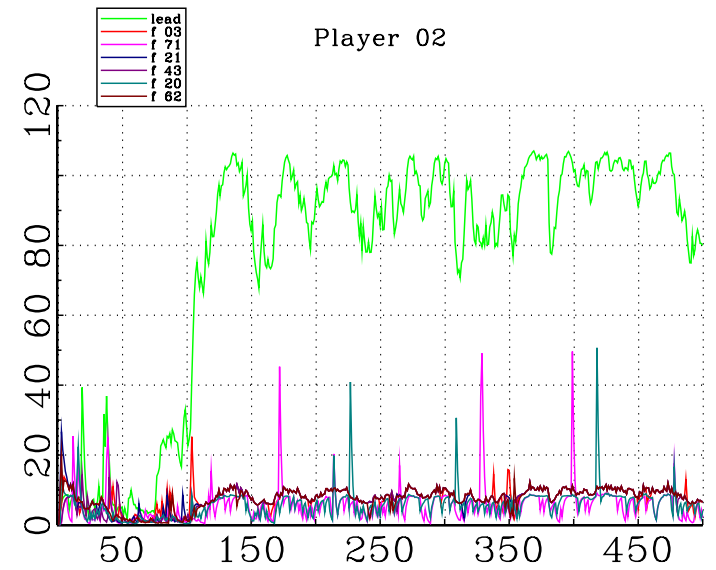

(c) Time-series $A_{0}-A_{6}$ for the eventual leader.

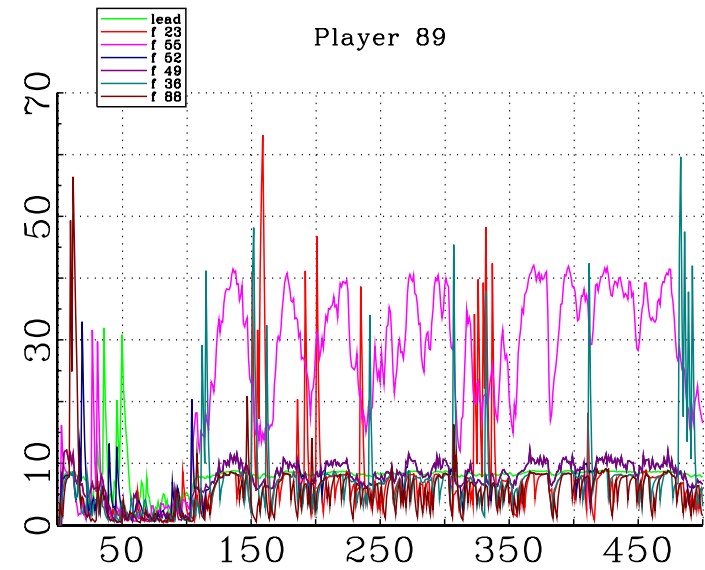

(e) Time-series $A_{0}-A_{6}$ for an agent two links from the leader.

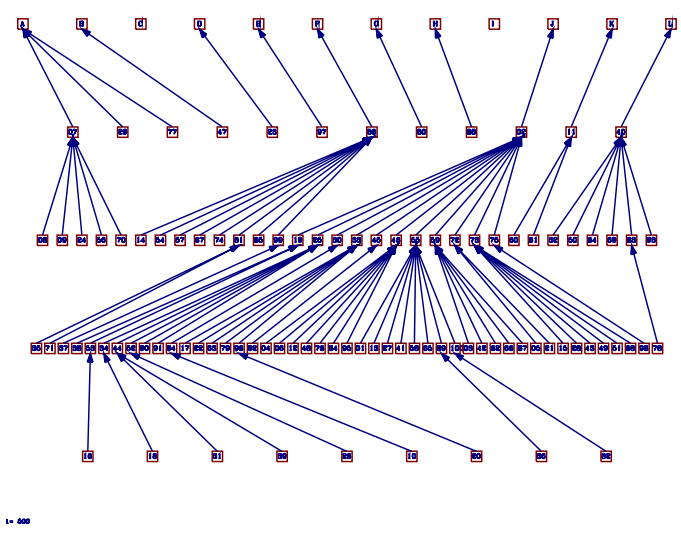

(b) Social structure in the final period $(T=500)$.

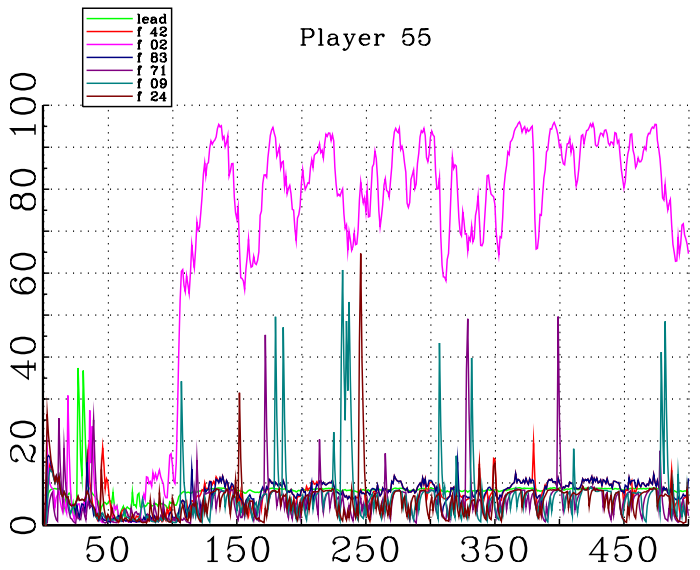

(d) Time-series $A_{0}-A_{6}$ for an agent one link from the leader.

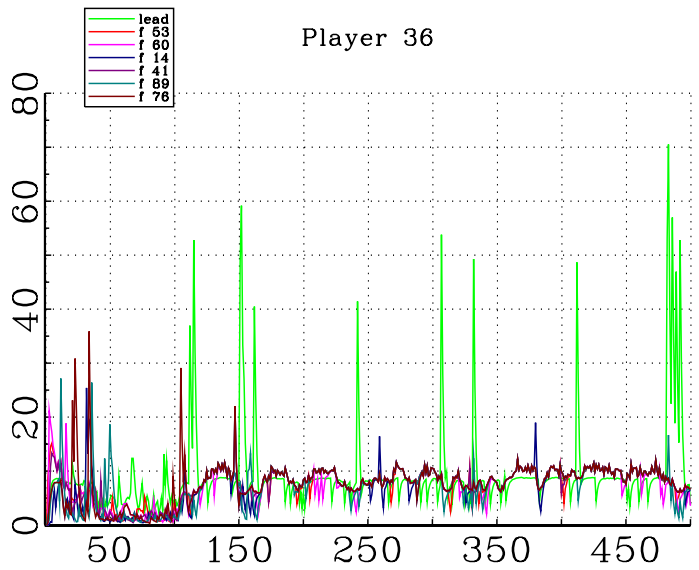

(f) Time-series $A_{0}-A_{6}$ for an agent most distant from the leader.

Figure 13: Strong lead: $B=-0.055$ and $n_{s}^{*}=15$. In large conformity populations, the attraction to lead is greater than that of conformity. 


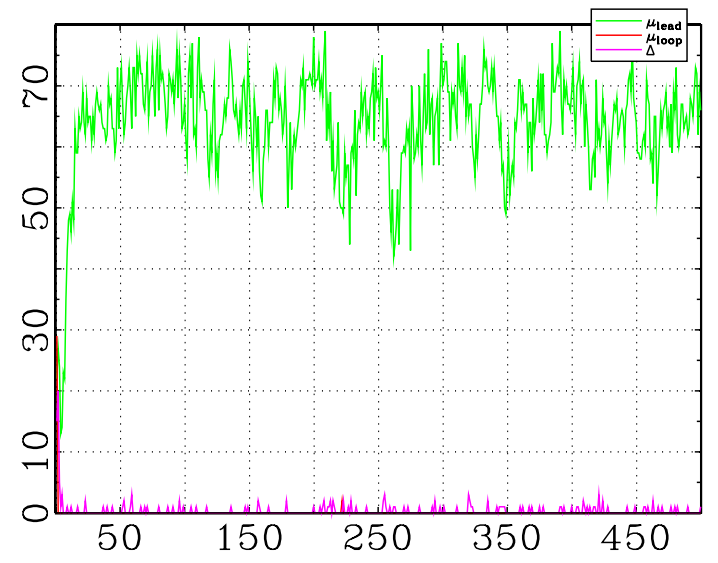

(a) Time-series of $\left\{\mu_{\text {lead }}, \mu_{\text {loop }}, \Delta\right\}=\{$ number lead, number looped, inefficiency $\}$

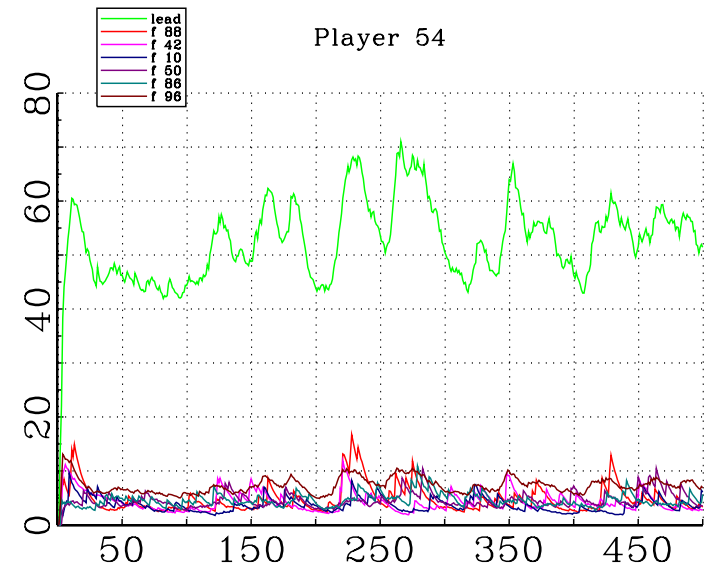

(c) Time-series $A_{0}-A_{6}$ for the eventual leader.

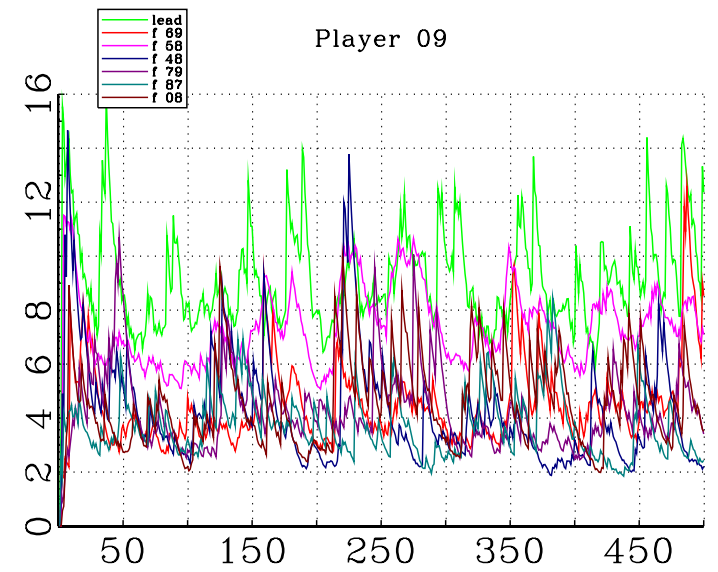

(e) Time-series $A_{0}-A_{6}$ for an agent two links from the leader.

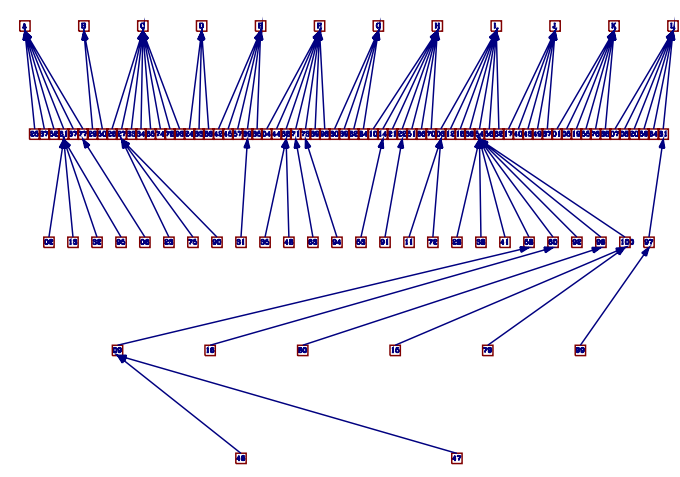

(b) Social structure in the final period $(T=500)$.

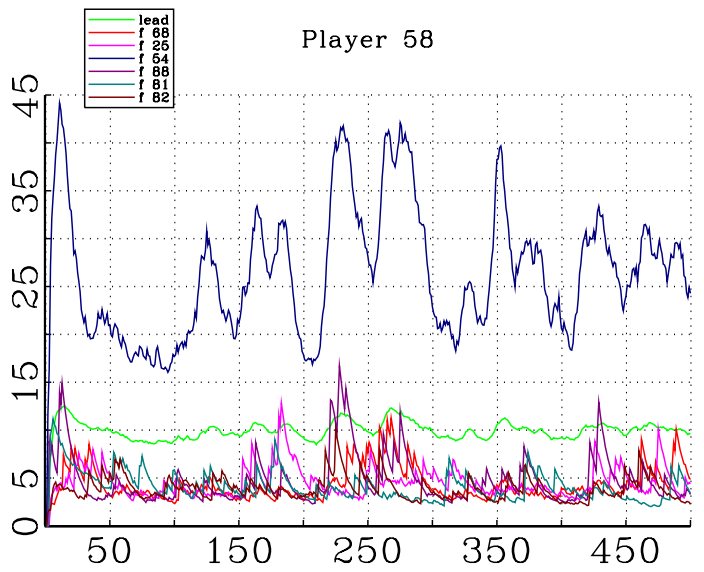

(d) Time-series $A_{0}-A_{6}$ for an agent one link from the leader.

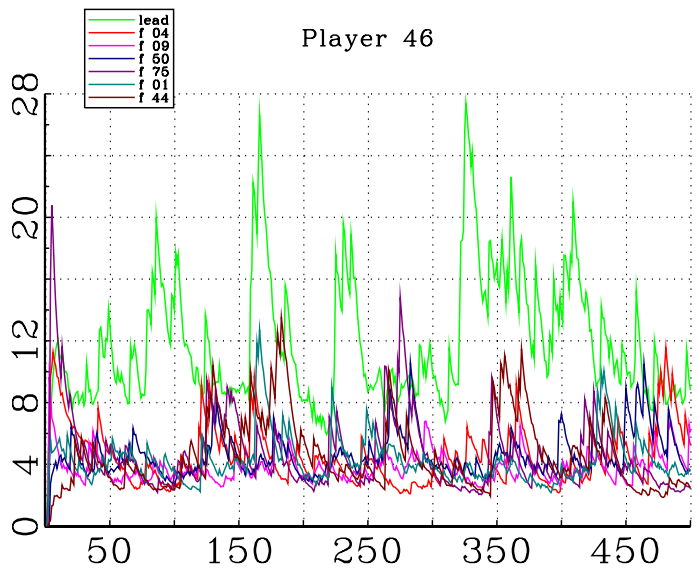

(f) Time-series $A_{0}-A_{6}$ for an agent most distant from the leader.

Figure 14: Strong lead with lock-in: $B=-0.055, \kappa=0.2$ Lock-in disrupts the mixed-strategy equilibrium supporting a $n_{s}>n_{s}^{*}$ structure by increasing discernability, to the population's detriment. 


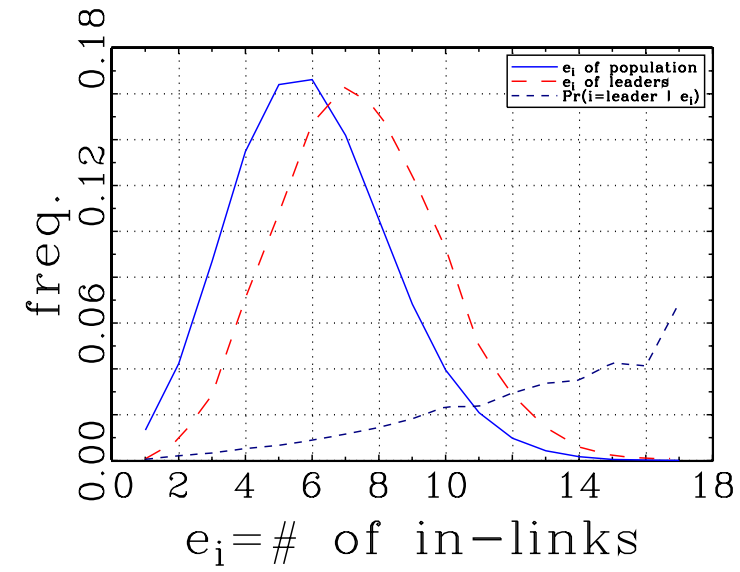

(a) Leader in-link distribution under quick identification and emergence

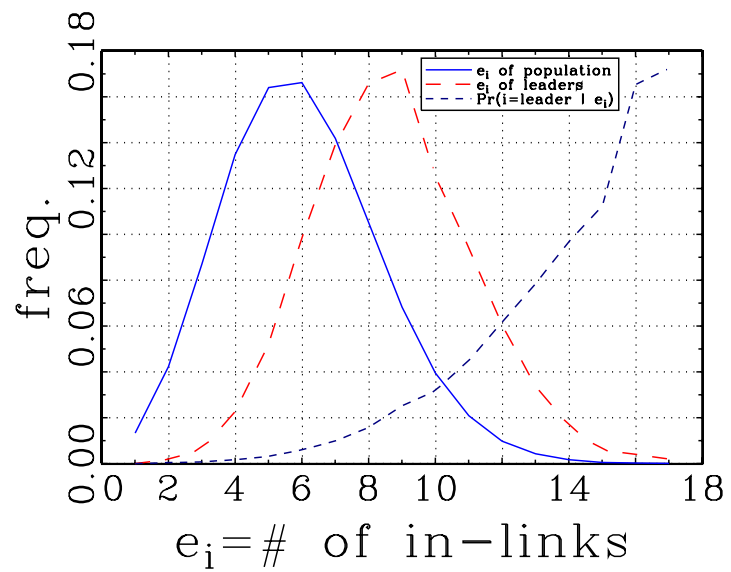

(b) Leader in-link distribution under slow identification and emergence

Figure 15: Distribution of $e_{i}$ among the general agent population (solid blue) and among dominant leaders (long-dashed red). The conditional probability of leading (short-dashed black) increases in $e_{i}$.

Table 3: Probability of becoming leader (5,000 iterations). Std err in parenthesis.

\begin{tabular}{|c|c|c|c|c|c|c|}
\hline Treatment & $\bar{e}_{i}$ & $\bar{e}_{\text {leaders }}$ & $\operatorname{Pr}($ leader & $\beta_{\text {inlink }}$ & instances leader- $\bar{e}_{i}=1$ & rel. freq. leader- $\bar{e}_{i} \geq 17$ \\
\hline \hline Quick & 6 & 7.4 & 0.01 & $0.0026^{*}$ & 4 of 6655 & 0.069 \\
& & & & $(5.95 e-5)$ & & \\
\hline Slow & 6 & 8.7 & 0.01 & $\begin{array}{c}0.0048^{*} \\
(5.92 e-5)\end{array}$ & 0 of 6655 & 0.172 \\
& & & & & \\
\hline
\end{tabular}

* Significance at the $1 \%$ level.

The treatment presented in Figure 15b sets $A_{0,0}=35$, ensuring that every agent leads in the first period. The in-links, thus, have no impact on first period payoffs. In addition, the IOC parameter is reduced to $\mu=0.2$ to slow down the evolutionary process. With $\rho_{i, 0}=1$ there is no advantage to be gained from the initial structure. All advantage arises out of the greater visibility as individuals develop preferences based on experience and observation. Apparently, the slower building of a reputation enhances the advantage of visibility.

Table 3 reports indicative statistics for the two treatments. The reported $\beta_{\text {inlink }}$ is the coefficient on a simple OLS projection of whether agent $i$ led on the number of inlinks in excess of six. The unconditional probability of leading is also the regression intercept. Notice that, at least for the quick treatment, visibility does not completely dominate luck as even an agent with only one observer can still emerge as leader (for the slow treatment, nine of 21,124 agents with only two observers emerge as leader). 
Table 4: The population is distributed over a symmetric network in which each agent has $n_{d}=6$ outward directed links and $n_{d}=6$ inward directed links with no overlap between in- and out-linked neighbors. Homogeneous treatment: each agent employs the same EWA and logit parameters in responding to personal experiences. Agent 1 always leads in the alternate. The total instances as leader is the sum for the individual over the 5,000 iterations.

\begin{tabular}{|c|c|c|c|c|c|}
\hline & \multirow{6}{*}{ Agent 1 } & Avg & Std & Min & Max \\
\cline { 3 - 7 } & Runs as the leader \\
\hline \hline \multicolumn{7}{|c|}{ Avg. earnings } \\
\hline Homogeneous population & 46 & 50.04 & 7.18 & 35 & 68 \\
\hline Agent 1 always leads & 605 & 44.39 & 9.79 & 22 & 64 \\
\hline \multicolumn{7}{|c|}{ Avents 2 - 100 } \\
\hline Homogeneous population & 32.21 & 32.21 & 0.32 & 31.29 & 32.92 \\
\hline Agent 1 always leads & 15.88 & 30.76 & 2.11 & 27.35 & 34.26 \\
\hline
\end{tabular}

5000 iterations, $T=100$.

\subsubsection{Leading behavior}

A dominant leader is someone who both leads and attracts followers. The agent cannot be assured of the latter but does have control over the former. If $x$ is the number of times an agent becomes the leader after $q$ iteration runs of the simulation, then for a homogeneous population of size $n$ on a symmetric network, $x \sim B(q, 1 / n)$. For 5,000 iterations and $n=100, E(x)=50$ and $\operatorname{SD}(x)=7.04$. The first row of Table 4 reports the statistics produced from 5,000 iterations.

Consider $n$ agents distributed over a symmetric social network of which $n-1$ are homogeneous in their use of the EWA process to guide actions. Agent 1 fails to adjust, choosing to always lead. Agent 1 emerges as the dominant leader in 605 of 5,000 iterations or just over $12 \%$ of the time. With agent 1 over-represented in instances as the dominant leader, the distribution of the number of observations as the dominant leader shifts down for agents 2 through 100. While agent 1 experiences great success in becoming the leader, she suffers in terms of earnings. The foregone income from failing to follow the $88 \%$ of the time when someone else emerges as the leader exceeds the benefit gained from the increased probability of leading.

The experience of agent 1 suggests that one can, through strategic behavior, influence the evolutionary process and the outcome. Such behavior also impacts the other agents of the population, not just in the lost opportunities to lead, but also through the impact agent 1's behavior has on the structures that tend to emerge depending on her success or failure to be leader. Those on agent 1's contact list, and thus dependent on agent 1 to help build a following, suffer. The six agents on agent 1's contact list lead on average 30.7 times with the most successful leading only 33 times, well below the average of $44 .^{8}$ Those who can directly observe agent 1 benefit from the discretion to follow her when she is leader optimize on other options when

\footnotetext{
${ }^{8} 17$ of 99 agents lead 33 times of less.
} 


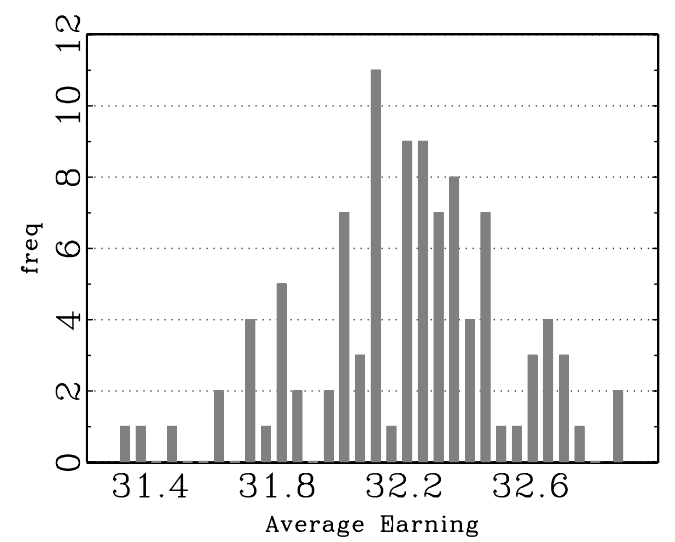

(a) Baseline: Homogeneous population with agent 1 updating behavior based on experiences

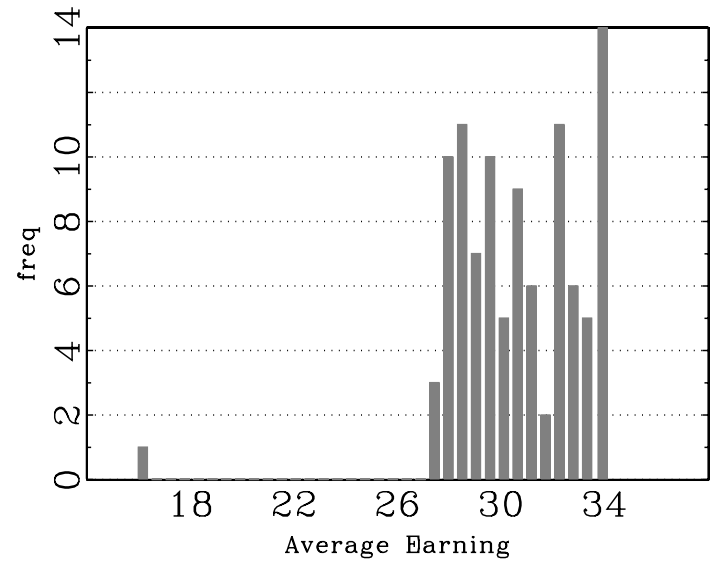

(b) Treatment: Agent 1 always leads, all others update probabilities according to the EWA.

Figure 16: Distribution of per-period individual earnings (5,000 iterations). The population is distributed over a symmetric directed network with no two agents able to directly observe each other. The behavior increases the probability that agent 1 emerges as the dominant leader of the simulation but lowers agent 1 's expected earnings. The distribution of earning for agents 2 - 100 increases in variance relative to the base.

she does not. The average mean earning among those observing agent 1 is 33.8 with the lowest observed mean earnings at 33.3 , well above the average of $27 .{ }^{9}$ Figure 16 presents the distribution of the per period average earning by each agent.

The diminished average earnings experience by agent 1 reflect, in part, her continuing to lead after the entire population has formed a hierarchy under another agent. It is possible that agent 1 can profitably persist in leading until such point that doing so is no longer advantageous. Such a strategy can be approximated by incrementally increasing an individual's $A_{0,0}(i)$, the starting value for the lead performance measure. With $A_{0,0}(j)=35, j \in\{2, \ldots, 100\}$, Figure 17 reveals a rise and then fall in agent 1's average earning relative to that of the population as $A_{0,0}(1)$ increases. From a below average earning with $A_{0,0}(1)=1$, agent 1 's average earnings increase as she more persistently pursues the lead strategy. Agent 1's average earnings is observed above the 95 percentile of the population at $A_{0,0}(1)=150$ and peaks at $A_{0,0}(1)=250$ with the highest average earnings.

\subsection{Contact Selection}

Allowing individuals in the population to replace low performing contacts with new contacts and the hierarchy collapses the vertical tree structure as followers find more direct imitation paths to the leader. Eventually a nearly completely horizontal structure emerges with the entire follower population directly imitating the

\footnotetext{
${ }^{9} 33.3$ is the 18 th highest mean earning of agents $2-100$.
} 


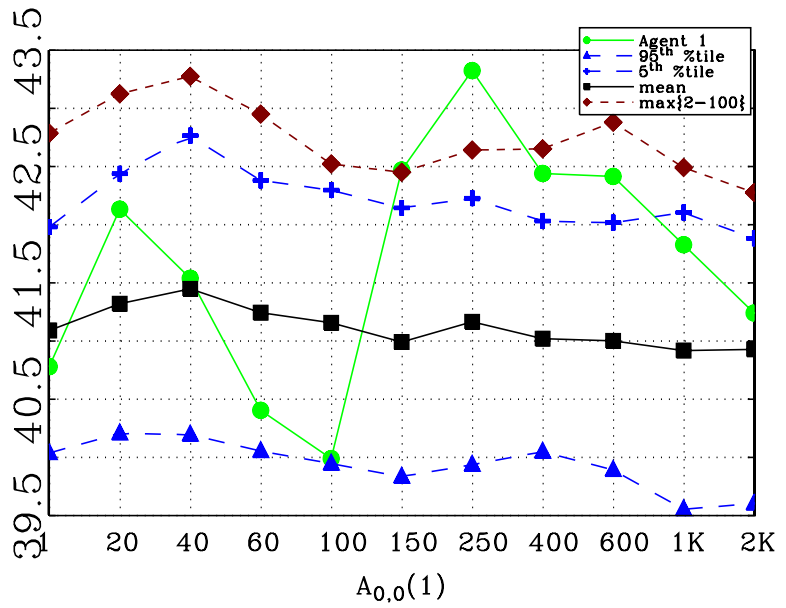

Figure 17: $B=1.21, \mu=0.2, \lambda=0.8, \phi_{l}=\phi_{f}=0.9, \kappa_{l}=\kappa_{f}=0, \delta_{l}=0.9, \delta_{f}=1, A_{0,0}(i \neq 1)=35$, $T=100,1,000$ iterations for each $A_{0,0}(1)$. Average earning of agent 1 relative to the population as agent 1 increases persistence in leading. Population mean average earning and $10 \%$ bands shown. Also the maximum average earnings excluding agent 1.

dominant leader . The payoff to the leader is unchanged. The same is true for those who were most distant from the dominant leader according to the initial contact structure. Those agents originally at intermediate distances from the leader see their payoff diminished by the collapse of the vertical structure as they lose their distance advantage over others in the population.

The simulation producing Figure 18 allows individuals to drop the lowest performing contacts with $w_{i, t}^{j}<\underline{w}_{i}$ with $\underline{w}_{i}=.5\left(1-\rho_{i}\right) / d$. Under this criterion, the contact is dropped if he or she substantially underperforms with a probability weight that is only $50 \%$ of the average for all contacts.

\section{Conclusion}

When the consumers are more concerned with the social phenomenon of a product than with the product itself, a leader plays a vital role in coordinating the population. Individuals have an interest in their position in the social structure when leading the population earns a premium reward. Despite the inherent inequality in the outcome, simulations indicate that a population can, when employing an appropriate mechanism of adjustment, organize itself into the equilibrium social structure that benefits all. The self-serving but myopic adaptive behavior produced by the experience weighted attractor (EWA) generates the needed adjustments in individual behavior that places the larger population into the role of follower behind a single emergent leader. That the emergence takes place with individuals unaware of the larger social structure addresses the 


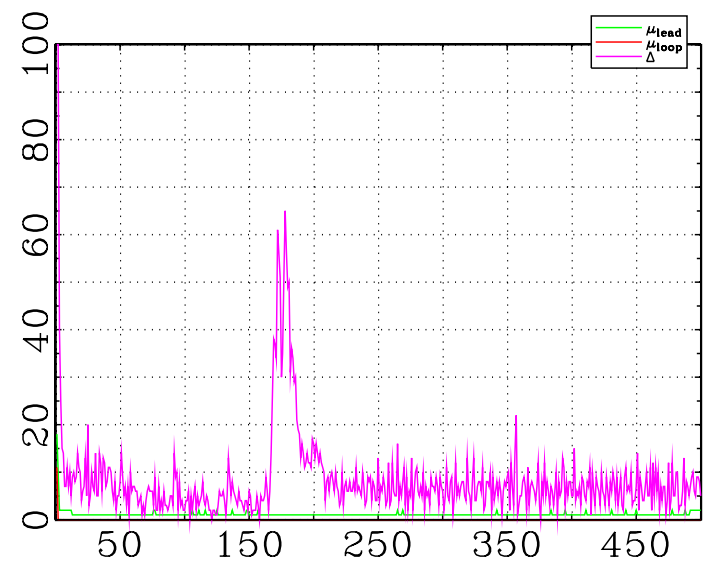

(a) Time-series of $\left\{\mu_{\text {lead }}, \mu_{\text {loop }}, \Delta\right\}=\{$ number lead, number looped, inefficiency $\}$

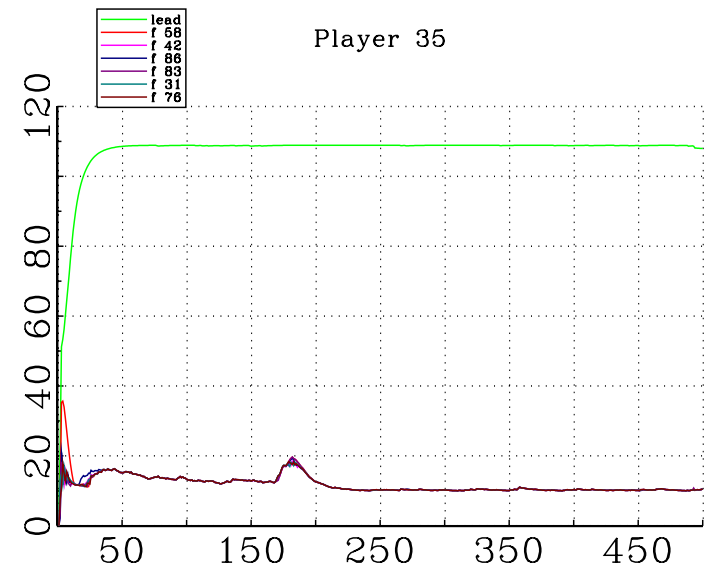

(c) Time-series $A_{0}-A_{6}$ for the eventual leader.

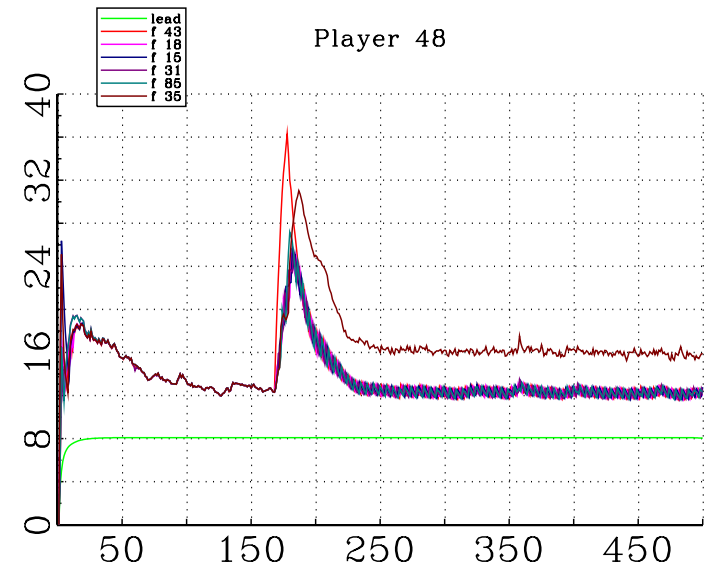

(e) Time-series $A_{0}-A_{6}$ for an agent initially two links from the leader.

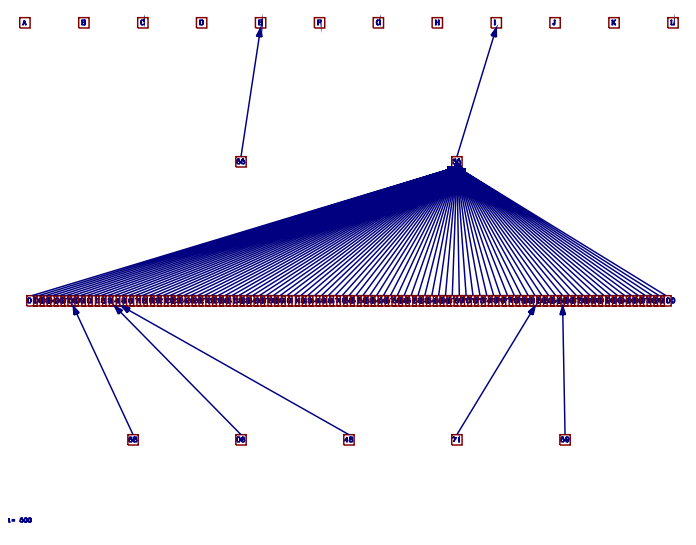

(b) Social structure in the final period $(T=500)$.

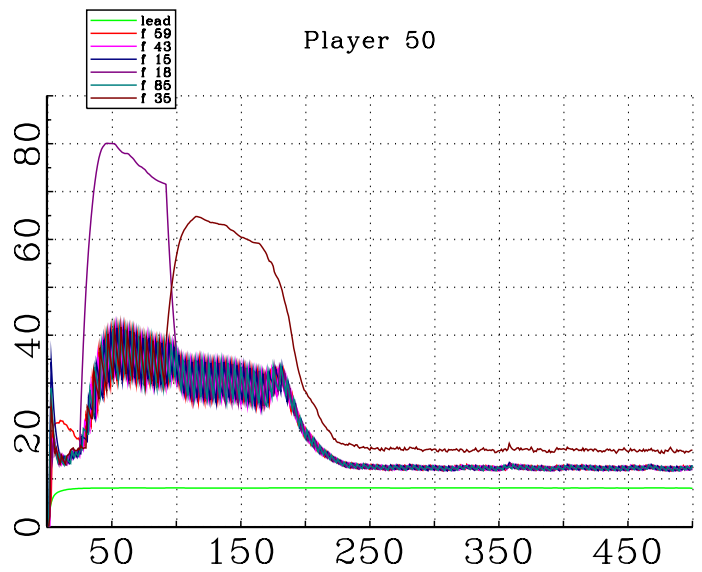

(d) Time-series $A_{0}-A_{6}$ for an agent initially one link from the leader.

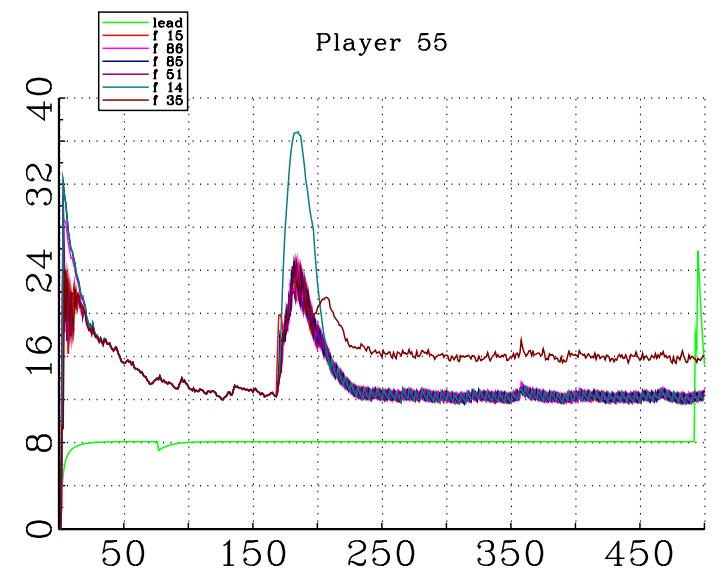

(f) Time-series $A_{0}-A_{6}$ for an agent who leads in $T$.

Figure 18: With the ability to unilaterally and costlessly establish new potential imitation links, agents eventually find a direct link to the dominant leader. With small but non-zero probability, each follower follows someone other than the leader such that there is typically a thinly populated second tier. 
Kirman et al. (2007) criticism that endogenous network models tend to require unreasonably high knowledge regarding the full social network.

The failure in the population to organize under low intensity of choice conditions or when agents downweight the performance signal of untried strategies points to the key elements necessary for structure to emerge. Outcomes early in the simulation are primarily driven by random events. In the absence of any accommodation to these early random events, they become transitory, subsumed by different randomized outcomes in the following period. Emergence of order requires adaptation that transforms early transitory outcomes into permanent components of a social structure. When agent $i$ is initially lucky in selecting an option that is popular, this attracts the attention of those who can observe agent $i$, increasing their likelihood of imitating $i$ and thereby increasing the likelihood of a positive outcome for $i$ in subsequent periods. Agent i's success builds over time as the social structure adapts to, and thereby reinforces, her success.

The simulations identified locally stable mixed-strategy disequilibria structures. The disequilibria are supported by non-degenerative probability assigned to inferior choice options. The simulations also identified a Pareto improving mixed-strategy equilibrium that supports conformity enabling structures despite incentives that, in a pure strategy setting, undermine conformity by offering a strong reward to leading.

The backward-looking adaptive accommodation by the agent to her own evolving environment, governed by the EWA precludes the type of forward-looking strategic behavior an agent might attempt in order to influence the formation of the social structure to her own advantage. Personally advantageous enacted influence on the emergent social structure through forward looking strategic behavior is practiced to the detriment of the larger population. Strategic behavior in the form of deciding how long to lead before implementing the first follow impacts upon both individual and aggregate outcomes. 


\section{References}

Acemoglu, D., Bimpikis, K., Ozdaglar, A. 2010. Dynamics of information exchange in endogenous social networks. Working Paper 16410, National Bureau of Economic Research.

Acemoglu, D., Como, G., Fagnani, F., Ozdaglar, A. 2013. Opinion fluctuations and disagreement in social networks. Mathematics of Operations Research, 38, 1-27.

Ali, S. N., Kartik, N. 2012. Herding with collective preferences. Economic Theory, 51, 601-626.

Arifovic, J., Eaton, B. C., Walker, G. 2015. The coevolution of beliefs and networks. Journal of Economic Behavior \& Organization, 120, 46 - 63.

Bala, V., Goyal, S. 2000. A noncooperative model of network formation. Econometrica, 68, 1181-1229.

Banerjee, A. V. 1992. A simple-model of herd behavior. Quarterly Journal of Economics, 107, 797-817.

Blume, L. E., Durlauf, S. N. 2001. The interactions-based approach to socioeconomic behavior. In S. N. Durlauf, H. P. Young eds. Social Dynamics, Cambridge MA, MIT Press, 15-44.

Branch, W., McGough, B. 2008. Replicator dynamics in a cobweb model with rationally heterogeneous expectations. Journal of Economic Behavior \& Organization, 65, 224-244.

Brock, W. A., Durlauf, S. N. 2001. Discrete choice with social interactions. The Review of Economic Studies, $68,235-260$.

Camerer, C., Ho, T. H. 1999. Experience-weighted attraction learning in normal form games. Econometrica, $67,827-874$.

Chang, M. H., Harrington, J. E. 2007. Innovators, imitators, and the evolving architecture of problem-solving networks. Organization Science, 18, 648-666.

Corazzini, L., Pavesi, F., Petrovich, B., Stanca, L. 2012. Influential listeners: An experiment on persuasion bias in social networks. European Economic Review, 56, 1276-1288.

Crawford, V. P., Haller, H. 1990. Learning how to cooperate: Optimal play in repeated coordination games. Econometrica, 58, pp. 571-595.

DeGroot, M. H. 1974. Reaching a consensus. Journal of the American Statistical Association, 69, 118-121. 
Dutta, B., Jackson, M. O. 2000. The stability and efficiency of directed communication networks. Review of Economic Design, 5, $251-272$.

Ellison, G., Fudenberg, D. 1995. Word-of-mouth communication and social-learning. Quarterly Journal of Economics, 110, 93-125.

Goldbaum, D. 2016. Conformity and influence. SSRN Working Paper 2761711.

Hausman, J., McFadden, D. 1984. Specification tests for the multinomial logit model. Econometrica: Journal of the Econometric Society, 1219-1240.

Jackson, M. O., Wolinsky, A. 1996. A strategic model of social and economic networks. Journal of Economic Theory, 71, 44-74.

Katz, M. L., Shapiro, C. 1985. Network externalities, competition, and compatibility. American Economic Review, 75, 424-440.

Kirman, A., Ricciotti, R. F., Topol, R. L. 2007. Bubbles in foreign exchange markets. Macroeconomic Dynamics, 11, 102-123.

Schelling, T. C. 1971. Dynamic models of segregation. Journal of Mathematical Sociology, 1, 143-186.

Schelling, T. C. 1973. Hockey helmets, concealed weapons, and daylight savings - study of binary choices with externalities. Journal of Conflict Resolution, 17, 381-428.

Watts, A. 2001. A dynamic model of network formation. Games and Economic Behavior, 34, 331-341. 